\title{
ON STABILITY AND STRONG CONVERGENCE FOR THE SPATIALLY HOMOGENEOUS BOLTZMANN EQUATION FOR FERMI-DIRAC PARTICLES
}

\author{
XUGUANG LU AND BERNT WENNBERG
}

\begin{abstract}
The paper considers the stability and strong convergence to equilibrium of solutions to the spatially homogeneous Boltzmann equation for Fermi-Dirac particles. Under the usual cut-off condition on the collision kernel, we prove a strong stability in $L^{1}$-topology at any finite time interval, and, for hard and Maxwellian potentials, we prove that the solutions converge strongly in $L^{1}$ to equilibrium under a high temperature condition. The basic tools used are moment production estimates and the strong compactness of collision gain term. Key words: Boltzmann Equation, Fermi-Dirac particles, stability, strong convergence, equilibrium.
\end{abstract}

\section{INTRODUCTION AND MAIN RESULTS}

The classical Boltzmann equation, describes the evolution of a phase space density of particles, under the assumption that they only interact by pairwise (elastic) collisions. Under suitable hypotheses it is then possible to derive the following equation,

$$
\frac{\partial}{\partial t} f+v \cdot \nabla_{x} f=\iint_{\mathbb{R}^{3} \times S^{2}} B\left(v-v_{*}, \omega\right)\left\{f^{\prime} f_{*}^{\prime}-f f_{*}\right\} d \omega d v_{*} \cdot
$$

Here $f=f(x, v, t) \geq 0$ is a density in phase space; normally $x \in \Omega \subset \mathbb{R}^{3}$, and $v \in \mathbb{R}^{3}$. In the collision integral in the right hand side, $f^{\prime}$ denotes $f\left(x, v^{\prime}, t\right)$, et.c. where $\left(v^{\prime}, v_{*}^{\prime}\right)$ and $\left(v, v_{*}\right)$ denote the velocities of two particles before and after a collision. All this is described in more detail below.

From now on in this paper, only the spatially independent case is considered, and hence $f=f(v, t)$, and the term $v \cdot \nabla_{x} f$ disappears.

The right-hand side of (1.1), the collision operator, is quadratic, because the only interactions considered are binary collisions, and the corresponding equilibrium distribution is the Boltzmann-Maxwell distribution (centred around a mean velocity $u ; R$ is the gas constant),

$$
M(v, t)=\frac{\rho}{(2 \pi R T)^{3 / 2}} e^{-|v-u|^{2} / 2 R T}
$$

When quantum effects must be taken into account, then particles in equilibrium satisfy either Bose-Einstein statistics or Fermi-Dirac statistics. In the latter case, which is the only one studied in this paper, the Pauli exclusion principle holds. According to this, only one particle is allowed in each one of available quantum states, and then the equilibrium distribution is either of the form

$$
M(v)=F_{\varepsilon, a, b}(v):=\frac{a e^{-b\left|v-v_{0}\right|^{2}}}{a+\varepsilon e^{-b\left|v-v_{0}\right|^{2}}}, \quad \varepsilon, a, b>0,
$$

or a characteristic function

$$
M(v)= \begin{cases}c & |v|<a \\ 0 & \text { otherwise }\end{cases}
$$

A modification of the Boltzmann equation that takes into account the Pauli exclusion principle is

$\frac{\partial}{\partial t} f(v, t)=\iint_{\mathbb{R}^{3} \times S^{2}} B\left(v-v_{*}, \omega\right) \times\left\{f^{\prime} f_{*}^{\prime}(1-\varepsilon f)\left(1-\varepsilon f_{*}\right)-f f_{*}\left(1-\varepsilon f^{\prime}\right)\left(1-\varepsilon f_{*}^{\prime}\right)\right\} d \omega d v_{*}$.

Here $\varepsilon=(h / m)^{3} / g$, where $\mathrm{h}$ is the Planck constant, and $m$ and $g$ are the mass and "statistical weight" of a particle. In equation (1.4), the factor $(1-\varepsilon f)$ is a ratio which decreases the probability that two FermiDirac particles share a small common velocity region. From a mathematical point of view, one can easily normalise the equation in such a way that $\varepsilon=1$, and most of the results of this paper are fully independent of such a rescaling. However, one of our main results, Theorem 2, states that, when no rescaling is carried 
out, then the solutions of (1.4) converge to solutions of (1.1) as $\varepsilon \rightarrow 0$, and hence we keep the factor $\varepsilon$ in most cases.

This modified Boltzmann equation is from now on called the Boltzmann-Fermi-Dirac equation (or Boltzmann-FD equation).

The physical properties of equation (1.4), and its derivation, are discussed e.g. in [11], chapter 17. A nice review of properties of quantum and relativistic versions of the Boltzmann equation, as well as new results on for example equilibrium distributions et.c. is [16]. The classification of equilibria to (1.4) was obtained in [20]; of course the importance of the Fermi-Dirac distribution was recognised much earlier (see e.g. [26]).

In order to proceed, we define, in turn

$$
\begin{array}{ll}
M_{0}(f(\cdot, t))=\int_{\mathbb{R}^{3}} f(v, t) d v, & v_{0}=\frac{1}{M_{0}} \int_{\mathbb{R}^{3}} f(v, t) v d v, \\
M_{2}(f(\cdot, t))=\int_{\mathbb{R}^{3}} f(v, t)\left|v-v_{0}\right|^{2} d v . &
\end{array}
$$

and then the temperature $T$ of the gas, and the Fermi-temperature, $T_{F}$ (see [11] and [28], page 43-44):

$$
T=\frac{m}{3 k_{B}} \cdot \frac{M_{2}\left(f_{0}\right)}{M_{0}\left(f_{0}\right)}, \quad T_{F}=\left(\frac{3 M_{0}\left(f_{0}\right)}{4 \pi \mathrm{g}}\right)^{2 / 3} \cdot \frac{h^{2}}{2 m k_{B}} ;
$$

here $k_{B}$ is the Boltzmann constant, and $h, m$ and $g$ are the Planck constant, the particle mass, and the statistical weight, as above.

The temperature plays a different role in the quantum case as compared to the classical case: because of the Pauli principle, the density is limited by $1 / \varepsilon$ (this guarantees that the factor $(1-\varepsilon f)$ remains nonnegative), and hence the temperature is bounded from below:

$$
T \geq \frac{2}{5} T_{F}
$$

In summary, the main results of this paper are as follows. We assume the particles interact by hard cutoff potentials or Maxwellian cut-off potentials. The norms used are $\|f\|_{L_{s}^{1}}=\int_{\mathbb{R}^{3}}|f(v)|\left(1+|v|^{2}\right)^{s / 2} d v$

- Theorem 1 (strong stability with respect to initial data): Let $f(v, t)$ and $g(v, t)$ be solutions to the Boltzmann-Fermi-Dirac equation (1.4) with initial data $f_{0}$ and $g_{0}$, and assume that these satisfy bounds on mass and energy, as well as $0 \leq f_{0}, g_{0} \leq 1 / \varepsilon$. There is an increasing function $\Phi_{f_{0}}$ : $\mathbb{R} \rightarrow \mathbb{R}$ (depending only on the initial data $f_{0}$ ), and a constant $c$ such that

$$
\sup _{t \geq 0} e^{-c t}\|f(t)-g(t)\|_{L_{2}^{1}} \leq \Phi_{f_{0}}\left(\left\|f_{0}-g_{0}\right\|_{L_{2}^{1}}\right) .
$$

- Theorem 2 (strong continuity with respect to $\varepsilon$ ): Let $f^{(\varepsilon)}(v, t)$ be the solution to (1.4), and $f(v, t)$ the solution to (1.1), with the same initial data. Then

$$
\sup _{t \geq 0} e^{-c t}\left\|f^{(\varepsilon)}(t)-f(t)\right\|_{L_{2}^{1}} \rightarrow 0, \quad(\varepsilon \rightarrow 0) .
$$

- Theorem 3 (strong convergence to equilibrium for the Boltzmann-Fermi-Dirac equation): There is a constant $\gamma(1)(>2 / 5)$, such that if the initial data $f_{0}$ to (1.4) have a sufficiently large temperature, $T\left(f_{0}\right) \geq \gamma(1) T_{F}$, then the solution $f(v, t)$ converges to the unique equilibrium state $F$ corresponding to $M_{0}\left(f_{0}\right)$ and $M_{2}\left(f_{0}\right)$ :

$$
\|f(t)-F\|_{L_{2}^{1}} \rightarrow 0 \quad(t \rightarrow \infty) .
$$

With this "high temperature condition" we obtain to a large extent the same results on stability and convergence for in this case as for the classical Boltzmann equation (se e.g. Wennberg [25], Carlen and Carvalho [6], Toscani and Villani[23], Abrahamsson [1], or a detailed review by Villani [29] ), and with Theorem 1 the earlier stability results for the classical Boltzmann equation are also improved.

We also prove prove for all temperatures that $\|f(t)-F\|_{L_{2}^{1}}$ is in a sense non-expanding, and we believe that the high temperature condition in Theorem 3 is only a technical condition, but it is needed in our proof of convergence to equilibrium. The importance of a condition on temperature is observed in for example [14], [15] and [21]. The non-expansitivity of $\|f(t)-F\|_{L_{2}^{1}}$ bears resemblance with the use of the 
Csiszár-Kullback inequality in connection with the kinetic theory of gases (e.g. [27] or [9] and references therein).

The details of Theorem 2, and its proof is given i Section 2, and Theorem 3 is treated in Section 3; the remaining part of this section is devoted to some notation, and som background material on the Boltzmann equation for Fermi-Dirac particles.

Though it is relevant to consider relativistic velocities in combination with the quantum versions of the Boltzmann equation (see [16]), we consider only small velocities here, and hence the velocities before and after a collision are related by

$$
v^{\prime}+v_{*}^{\prime}=v+v_{*}, \quad\left|v^{\prime}\right|^{2}+\left|v_{*}\right|^{2}=|v|^{2}+\left|v_{*}\right|^{2}
$$

which are equivalent to a family of orthogonal linear transforms $\left(v, v_{*}\right) \mapsto\left(v^{\prime}, v_{*}^{\prime}\right)$ :

$$
v^{\prime}=v-\left\langle v-v_{*}, \omega\right\rangle \omega, \quad v_{*}^{\prime}=v_{*}+\left\langle v-v_{*}, \omega\right\rangle \omega, \omega \in S^{2} .
$$

Here $S^{2}$ and $\langle\cdot, \cdot\rangle$ denote the unit sphere and the inner product in $\mathbb{R}^{3}$.

In (1.1) as well as in (1.4), $B(z, \omega)$ is a non-negative Borel-function that depends on $|z|$ and on $|\langle\cdot, \cdot\rangle|$ only. Its gives the rate at which a given combination of in-going velocities results in a given set of outgoing velocities; for classical particles its exact form can be derived, at least in the case of repulsive forces corresponding to inverse power laws. For Fermi-Dirac particles it is not quite so clear, and in particular applications (for example semi-conductors), the velocity space is not $\mathbb{R}^{3}$, but periodically repeated Brillouin zones. Letting the velocity vary over the full $\mathbb{R}^{3}$ as we do here, corresponds to the "parabolic band approximation" used in semi-conductor theory. Here we assume exactly the same form for $B$ in both cases:

$$
B(z, \omega)=b(\theta)|z|^{\beta}, \quad z \in \mathbb{R}^{3}, \quad 0 \leq \beta \leq 1,
$$

where $\cos (\theta)=|\langle\cdot, \cdot\rangle| /|z|$, and $\beta$ is a constant, while $b(\theta)$ is strictly positive in $0<\beta<\pi / 2$. For $b(\theta)$ we also assume that

$$
0<A_{0}:=4 \pi \int_{0}^{\pi / 2} b(\theta) \sin (\theta) d \theta<\infty .
$$

This is Grad's cutoff condition, which is not satisfied for the inverse potentials mentioned above. We refer to [10] or [28] for the derivation of (1.8). The cutoff condition was introduced to simplify the mathematical treatment of (1.1), but now there is a theory also for the so-called non-cutoff case (see. Arkeryd [5], Goudon [17] and Villani [31]). A recent article by Alexandre and Villani, [2], treats also the space dependent case.

When (1.9) holds, the collision integral in (1.1) and in (1.4) (which we now denote by $Q(f)$ ) can be decomposed as a difference of the "gain term" $Q^{+}(f)$ and the "loss term" $Q^{-}(f)$ :

$$
\begin{aligned}
Q(f)(v) & =Q^{+}(f)(v)-Q^{-}(f)(v), \\
Q^{+}(f)(v) & =\iint_{\mathbb{R}^{3} \times S^{2}} B\left(v-v_{*}, \omega\right) f^{\prime} f_{*}^{\prime}(1-\varepsilon f)\left(1-\varepsilon f_{*}\right) \omega d v_{*}, \\
Q^{-}(f)(v) & =\iint_{\mathbb{R}^{3} \times S^{2}} B\left(v-v_{*}, \omega\right) f f_{*}\left(1-\varepsilon f^{\prime}\right)\left(1-\varepsilon f_{*}^{\prime}\right) d \omega d v_{*} .
\end{aligned}
$$

A solution of the Boltzmann-Fermi-Dirac equation is defined as follows. As usual, we consider the weighted $L^{1}$-spaces

$$
\begin{gathered}
L_{s}^{1}\left(\mathbb{R}^{3}\right)=\left\{f\left|\|f\|_{L_{s}^{1}\left(\mathbb{R}^{3}\right)}:=\int_{\mathbb{R}^{3}}\right| f(v) \mid\left(1+|v|^{2}\right)^{s / 2} d v<\infty\right\}, \\
\|f\|_{L_{s}^{1}\left(\mathbb{R}^{3}\right)} \equiv\|f\|_{L_{s}^{1}} .
\end{gathered}
$$

Assume that $B$ satisfies (1.8) and (1.9). Moreover, assume that the initial data $f_{0}(v)$ to eq.(1.4) satisfy

$$
0 \leq f_{0}(v) \leq 1 / \varepsilon, \quad f_{0} \in L_{2}^{1} .
$$


Then $f(v, t) \in L_{l o c}^{\infty}\left([0, \infty) ; L_{2}^{1}\right)$ is a solution to (1.4) if there is a null-set $Z \subset \mathbb{R}^{3}$ such that for all $v \in \mathbb{R}^{3} \backslash Z$, and all $t \in[0, \infty)$,

$$
\begin{aligned}
& \int_{0}^{t} Q^{+}(f)(v, t) d \tau<\infty, \quad \int_{0}^{t} Q^{-}(f)(v, t) d \tau<\infty, \\
& f(v, t)=f_{0}(v)+\int_{0}^{t} Q(f)(v, \tau) d \tau .
\end{aligned}
$$

If in addition $f$ conserves mass, momentum and energy, i.e. if

$$
\int_{\mathbb{R}^{3}} f(v, t) \psi(v) d v \equiv \int_{\mathbb{R}^{3}} f_{0}(v) \psi(v) d v, \quad t \in[0, \infty)
$$

for $\psi(v)=1, v$ or $|v|^{2}$, then we say that $f$ is a conservative solution.

For Fermi-Dirac-particles, the entropy is defined by

$$
S(f)=\frac{1}{\varepsilon} \int_{\mathbb{R}^{3}}[-(1-\varepsilon f) \log (1-\varepsilon f)-\varepsilon f \log (\varepsilon f)] d v ;
$$

it should be noted that $-(1-\varepsilon f) \log (1-\varepsilon)$ and $-\varepsilon f \log (\varepsilon f)$ are both nonnegative for all $f$ satisfying $0 \leq f \leq 1 / \varepsilon$. At least formally, the corresponding entropy dissipation identity is given by

$$
S(f(t))=S\left(f_{0}\right)+\int_{0}^{t} e(f(\tau)) d \tau, \quad t \in[0, \infty)
$$

where

$$
e(f)=\frac{1}{4} \iiint_{\mathbb{R}^{3} \times \mathbb{R}^{3} \times S^{2}} B\left(v-v_{*}, \omega\right) \Gamma\left(f^{\prime} f_{*}^{\prime}(1-\varepsilon f)\left(1-\varepsilon f_{*}\right), f f_{*}\left(1-\varepsilon f^{\prime}\right)\left(1-\varepsilon f_{*}^{\prime}\right)\right) d \omega d v_{*} d v,
$$

and where $\Gamma(\cdot, \cdot)$ is a nonnegative function defined on $[0, \infty) \times[0, \infty)$ by $\Gamma(a, b)=(a-b) \log \left(\frac{a}{b}\right)$ for $a>0, b>0$, and $\Gamma(a, b)=+\infty$ when $a>b=0$ or when $0=a<b$; and finally $\Gamma(a, b)=0$ for $a=b=0$. Here and below we always denote $f(t)=f(\cdot, t)$.

In a space-homogeneous setting, with no source terms, equilibrium solution is the same as a stationary solution to Eq.(1.4), and this is equivalent to saying that it is a solution of the following equation,

$$
F^{\prime} F_{*}^{\prime}(1-\varepsilon F)\left(1-\varepsilon F_{*}\right)=F F_{*}\left(1-\varepsilon F^{\prime}\right)\left(1-\varepsilon F_{*}^{\prime}\right) \quad \text { a.e. on } \mathbb{R}^{3} \times \mathbb{R}^{3} \times S^{2},
$$

combined with the conditions $F \in L^{1}\left(\mathbb{R}^{3}\right)\|F\|_{L^{1}} \neq 0$ and $0 \leq F \leq 1 / \varepsilon$ on $\mathbb{R}^{3}$; as noted above, this is either a Fermi-Dirac distribution (1.2) or a characteristic function, depending on the temperature (this is established in [20]).

Some additional properties of the solutions to (1.4) are the following (taken from Lu [20], but see also [16]). For the classical Boltzmann equation, a rather complete review of the state of the art is [33].

- When the temperature $T>\frac{2}{5} T_{F}$, the only equilibrium solution is given by (1.2). The coefficients $a$ and $b$ are determined by the moments $M_{0}$ and $M_{2}$ as defined in (1.5). Moreover, $T, T_{F}$ and the coefficient $a$ are related by

$$
T=\gamma\left(\frac{1}{\varepsilon a}\right) T_{F}
$$

where

$$
\gamma(x)=\frac{2}{3^{5 / 3}}\left(\int_{0}^{\infty} \frac{r^{4}}{1+x e^{r^{2}}} d r\right)\left(\int_{0}^{\infty} \frac{r^{2}}{1+x e^{r^{2}}} d r\right)^{-5 / 3}, x>0 .
$$

The function $\gamma$ is strictly increasing on $(0, \infty)$ with the $\operatorname{limits}_{x \rightarrow 0^{+}} \gamma(x)=\frac{2}{5}, \lim _{x \rightarrow \infty} \gamma(x)=$ $\infty$.

- The Boltzmann Fermi-Dirac equation has a conservative solution which satisfies the entropy identity (1.11). Moreover, if $\beta>0$ in (1.8), then for all $s>2$,

$$
\|f(t)\|_{L_{s}^{1}} \leq C_{s}\left(1+t^{-(s-2) / \beta}\right), \quad \forall t>0,
$$

where the coefficient

$$
0<C_{s}=C_{b(\cdot), \beta, s}\left(\left\|f_{0}\right\|_{L_{0}^{1}},\left\|f_{0}\right\|_{L_{2}^{1}}\right)<\infty
$$


depends only on $\left\|f_{0}\right\|_{L_{0}^{1}},\left\|f_{0}\right\|_{L_{2}^{1}}, \beta, s$ and the angular function $b(\cdot)$, and for fixed $b(\cdot), \beta$ and $s$, the function $(x, y) \mapsto C_{b(\cdot), \beta, s}(x, y)$ is continuous on $(0, \infty) \times(0, \infty)$.

If $\beta=0$ and if $f_{0} \in L_{s}^{1}\left(\mathbb{R}^{3}\right)$ for some $s>2$, then $\sup _{t \geq 0}\|f(t)\|_{L_{s}^{1}}<\infty$.

- If $T=\frac{2}{5} T_{F}$, then the conservative solution of (1.4) is the second type of equilibrium, the characteristic function (1.3).

If $T>\frac{2}{5} T_{F}$, and if $\beta>0$, then

$$
f(\cdot, t) \rightarrow F \quad(t \rightarrow \infty) \quad \text { weakly in } \quad L^{1}\left(\mathbb{R}^{3}\right)
$$

The weak convergence, (1.16), holds in general for the Boltzmann-FD equation for all $\beta$ with $-3<$ $\beta \leq 0$, if for some $t_{0}>0$,

$$
\sup _{t \geq t_{0}} \int_{|v|>R} f(v, t)|v|^{2} d v \rightarrow 0 \quad \text { as } \quad R \rightarrow \infty,
$$

i.e. if the the solution satisfies a "tightness of energy"; however, in this paper only the case $\beta \geq 0$ is considered.

The $L^{\infty}$-bounds that hold automatically for solutions to the Boltzmann-FD equation makes it comparably easy to prove the existence of solutions, also for spatially inhomogeneous solutions (Dolbeault [12], P.L.Lions [19]). However due to this factor, the two equilibria at very low temperatures are hardly possible to distinguished in $L^{1}$-topology , because

$$
\int_{\mathbb{R}^{3}}\left|F_{a, b}(v)-\frac{1}{\varepsilon} \mathbb{1}_{\left\{\left|v-v_{0}\right| \leq R\right\}}\right| d v \rightarrow 0 \quad \text { when } \quad 0<\frac{T}{T_{F}}-\frac{2}{5} \rightarrow 0 .
$$

(Here and below $\mathbb{1}_{A}$ denotes the indicator function for a set $A$ ). Therefore the study of strong convergence to equilibrium is more difficult than for the classical Boltzmann model Eq.(1.1), where the proof of convergence to equilibrium does not depend on the temperature, or not necessarily even on the entropy (see [1], [9], [27], or the review [32]).

The main components in the proofs of Theorems 1 and 2 are an efficient use of the collision invariants 1.7, and the moment estimates (1.14) and (1.15), and in the proof of Theorem 3, we use a generalised version of P.L. Lions' result on regularity for the gain term of the Boltzmann equation.

\section{Strong Stability estimates}

This section contains two stability results for the Boltzmann Fermi-Dirac equation. The first one states that the solutions are stable with respect to perturbations of the initial data, and this implies at the same time, of course, the uniqueness of solutions; the second deals with stability with respect to variations in parameter $\varepsilon$.

Three rather technical lemmas are needed for the proofs of the theorems, and we begin by stating these lemmas; the proofs are postponed until after the proofs of the main results.

Lemma 1. Let $\varepsilon>0$ be a constant, let $f=f(v), g=g(v)$ be real functions satisfying $0 \leq f(v), g(v) \leq$ $1 / \varepsilon$. Let $\phi(v)=1+|v|^{2}$. Then

$$
\begin{aligned}
& \left(f f_{*}\left(1-\varepsilon f^{\prime}\right)\left(1-\varepsilon f_{*}^{\prime}\right)-g g_{*}\left(1-\varepsilon g^{\prime}\right)\left(1-\varepsilon g_{*}^{\prime}\right)\right) \\
& \quad \times\left(\phi^{\prime} \mathbb{1}_{\left\{f^{\prime}>g^{\prime}\right\}}+\phi_{*}^{\prime} \mathbb{1}_{\left\{f_{*}^{\prime}>g_{*}^{\prime}\right\}}-\phi \mathbb{1}_{\{f>g\}}-\phi_{*} \mathbb{1}_{\left\{f_{*}>g_{*}\right\}}\right) \\
& \leq(f \phi)\left|f_{*}-g_{*}\right|+(f \phi)_{*}|f-g|+\varepsilon f f_{*}\left(\left|f^{\prime}-g^{\prime}\right| \phi_{*}^{\prime}+\left|f_{*}^{\prime}-g_{*}^{\prime}\right| \phi^{\prime}\right) .
\end{aligned}
$$

This kind of expression appears naturally when differentiating norms of the collision operator.

The second lemma deals with certain transformations of the gain term in the collision operator. It is taken from $\mathrm{Lu}$ [20], and we refer to that paper for the proof; it is in many ways similar to the "Cancellation lemma" that can be found in [3]. 
Lemma 2. Let $w(t)$ and $\Psi(r)$ be nonnegative Borel functions on $[0,1]$ and $[0, \infty)$ respectively. Let $W(z, \omega)=w\left(|z|^{-1}|\langle z, \omega\rangle|\right)$. Then for any nonnegative measurable function $f$ on $\mathbb{R}^{3}$ and for all $v \in \mathbb{R}^{3}$

$$
\begin{aligned}
& \iint_{\mathbb{R}^{3} \times S^{2}} W\left(v-v_{*}, \omega\right) \Psi\left(\left|v-v_{*}\right|\right) f\left(v^{\prime}\right) d v_{*} d \omega \\
&= 4 \pi \int_{0}^{\pi / 2} \frac{\sin (\theta) w(\cos \theta)}{\cos ^{3} \theta}\left\{\int_{\mathbb{R}^{3}} \Psi\left(\frac{\left|v-v_{*}\right|}{\cos \theta}\right) f\left(v_{*}\right) d v_{*}\right\} d \theta, \\
& \iint_{\mathbb{R}^{3} \times S^{2}} W\left(v-v_{*}, \omega\right) \Psi\left(\left|v-v_{*}\right|\right) f\left(v_{*}^{\prime}\right) d v_{*} d \omega \\
&=4 \pi \int_{0}^{\pi / 2} \frac{\sin (\theta) w(\cos \theta)}{\sin ^{3} \theta}\left\{\int_{\mathbb{R}^{3}} \Psi\left(\frac{\left|v-v_{*}\right|}{\sin \theta}\right) f\left(v_{*}\right) d v_{*}\right\} d \theta .
\end{aligned}
$$

The third lemma, finally, is a point-wise estimate of moments of the gain term.

Lemma 3. Let $0 \leq k \leq 3$ be a constant, and let $0 \leq f \leq 1 / \varepsilon$ and $f \in L_{k+\beta}^{1}\left(\mathbb{R}^{3}\right)$ with $0 \leq \beta \leq 1$. Then for all $v \in \mathbb{R}^{3}$

$$
\varepsilon \iint_{\mathbb{R}^{3} \times S^{2}} B\left(v-v_{*}, \omega\right) f^{\prime} f_{*}^{\prime}\left(1+\left|v_{*}\right|^{2}\right)^{k / 2} d \omega d v_{*} \leq 2^{15} A_{0}\|f\|_{L_{k+\beta}^{1}}\left(1+|v|^{2}\right)^{\alpha / 2}
$$

where $\alpha=\max \{\beta,(k+\beta)(3-k) / 3\}$.

For the class of collision operators studied here, hard cut-off potentials, the main difficulty when studying stability is to control the behaviour at high velocities. To this end we introduce a "control function" $\Phi_{f_{0}}(r)$, defined for all non-negative functions $f_{0} \in L_{2}^{1}\left(\mathbb{R}^{3}\right)$ (see [23]):

$$
\Phi_{f_{0}}(r)=r+\sqrt{r}+\int_{|v|>\frac{1}{\sqrt{r}}} f_{0}(v)\left(1+|v|^{2}\right) d v, \quad r>0 ; \quad \Phi(0)=0 .
$$

Theorem 1. Let the kernel $B(z, \omega)$ be given by (1.8)-(1.4) with $0 \leq \beta \leq 1$. Let $\varepsilon \geq 0$ be a constant. For any given $0 \leq f_{0}, g_{0} \in L_{2}^{1}\left(\mathbb{R}^{3}\right)$ satisfying $\varepsilon f_{0} \leq 1, \varepsilon g_{0} \leq 1$, and $\left\|f_{0}\right\|_{L_{0}^{1}}>0$, let $f, g$ both be conservative solutions of Eq.(1.4) $(\varepsilon>0)$ or Eq.(1.1) $(\varepsilon=0)$ with initial data $\left.f\right|_{t=0}=f_{0}$, and $\left.g\right|_{t=0}=g_{0}$ respectively. Then for $\beta>0$

$$
\sup _{t \geq 0} e^{-c t}\|f(t)-g(t)\|_{L_{2}^{1}} \leq C \Phi_{f_{0}}\left(\left\|f_{0}-g_{0}\right\|_{L_{2}^{1}}\right)
$$

and for $\beta=0$

$$
\sup _{t \geq 0} e^{-c t}\|f(t)-g(t)\|_{L_{0}^{1}} \leq C\left\|f_{0}-g_{0}\right\|_{L_{0}^{1}}
$$

where $\Phi_{f_{0}}(\cdot)$ is defined by (2.3); the constants

$$
0<c=c_{b(\cdot), \beta}\left(\left\|f_{0}\right\|_{L_{0}^{1}},\left\|f_{0}\right\|_{L_{2}^{1}}\right), C=C_{b(\cdot), \beta}\left(\left\|f_{0}\right\|_{L_{0}^{1}},\left\|f_{0}\right\|_{L_{2}^{1}}\right)<\infty
$$

depend only on $\left\|f_{0}\right\|_{L_{0}^{1}},\left\|f_{0}\right\|_{L_{2}^{1}}, \beta$, and the angular function $b(\cdot)$. Especially, $c$ and $C$ do not depend on $\varepsilon$. Moreover for fixed $b(\cdot)$ and $\beta$, the functions $c_{b(\cdot), \beta}(x, y), C_{b(\cdot), \beta}(x, y)$ are continuous on $(x, y) \in$ $(0, \infty) \times(0, \infty)$

\section{Remarks.}

- For soft potentials $(-3<\beta<0)$, and under the same condition as in Theorem 1 for $\varepsilon>0$ (BFD model), it is easily proved that the estimate (2.5) still holds but in that case, the constants $c$ and $C$ depend on $\varepsilon$.

- In the proof of Theorem 1 , the exponent $(s-2) / \beta$ is essential. This was first written explicitly in [22], but it is implicit also in [25].

Proof of Theorem 1. The case $\beta=0$ is easy. Now suppose $\beta>0$. Let $f, g$ be the conservative solutions given in the theorem. We first assume that $f$ satisfies the moment production estimate (1.14)(1.15) (including the case $\varepsilon=0$ ). Since in our proof the moment estimate (1.14) is used only for $s=2+\beta$, the letter $C$ below always denotes different constants that have the property mentioned in this theorem. 
To prove (2.4) we can assume $\left\|f_{0}-g_{0}\right\|_{L_{2}^{1}}<1$. Let

$$
U_{\kappa}(t)=\|f(t)-g(t)\|_{L_{\kappa}^{1}}, \quad 0 \leq \kappa \leq 2 .
$$

Then following the same argument as given in [22, sect. 5], we need only to check the following estimates: for any $0<r \leq 1$

$$
\begin{array}{lll}
U_{2}(t) \leq U_{2}(0)+\frac{4}{\sqrt{r}} U_{1}(t)+C \Phi_{f_{0}}(r), & & t \in[0, r] \\
U_{1}(t) \leq U_{1}(0)+C \int_{0}^{t} U_{2}(\tau) d \tau, & & t \in[0, \infty) \\
U_{2}(t) \leq U_{2}(r)+C \int_{r}^{t}\left(1+\frac{1}{\tau}\right) U_{1}(\tau) d \tau, & & t \in[r, \infty) .
\end{array}
$$

Very briefly, Theorem 1 follows by the steps below (see [22] for details). $U_{1}(\cdot) \leq U_{2}(\cdot)$, and hence (2.8) and the Gronwall inequality imply that, for some constant $c$

$$
U_{2}(t) \leq U_{2}(1) e^{c(t-1)}, \quad t>1 .
$$

Next, from (2.7) and (2.8) one sees that, if $U_{2}(0)<r$,

$$
U_{2}(t) \leq \sup _{0 \leq \tau \leq r} U_{2}(\tau)+C r|\log (r)|+C \int_{0}^{t} U_{2}(\tau)|\log (\tau)| d \tau, \quad t \in[r, 1] .
$$

The same holds in the interval $0 \leq t<r$, and hence, by Gronwall again

$$
U_{2}(t) \leq\left(\sup _{0 \leq \tau \leq r} U_{2}(\tau)+C r|\log (r)|\right) e^{C t} .
$$

Finally, the inequalities (2.6) and (2.7) combine to

$$
\sup _{0 \leq \tau \leq r} U_{2}(\tau) \leq C\left(r+\sqrt{r}+\Phi_{f}(r)\right)
$$

and once can easily conclude from this.

Proof of (2.6): Let $\phi(v)=1+|v|^{2}$. By the integral equation (1.10), we have for a null set $Z \subset \mathbb{R}^{3}$,

$$
f(v, t) \geq f_{0}(v)-\int_{0}^{t} d \tau \iint_{\mathbb{R}^{3} \times S^{2}} B\left(v-v_{*}, \omega\right) f f_{*} d \omega d v_{*}, \quad v \in \mathbb{R}^{3} \backslash Z, t \geq 0 .
$$

This together with the conservation of mass and energy implies that

$$
\begin{aligned}
\int_{|v|>1 / \sqrt{r}} f(v, t) \phi(v) d v & =\int_{\mathbb{R}^{3}} f(v, t) \phi(v) d v-\int_{|v| \leq 1 / \sqrt{r}} f(v, t) \phi(v) d v \\
\leq & \int_{\mathbb{R}^{3}} f_{0}(v) \phi(v) d v-\int_{|v| \leq 1 / \sqrt{r}} f_{0}(v) \phi(v) d v \\
& +A_{0} \int_{0}^{t} d \tau \int_{|v| \leq 1 / \sqrt{r}} \int_{\mathbb{R}^{3}} f(v, \tau) \phi(v) f\left(v_{*}, \tau\right)\left|v-v_{*}\right|^{\beta} d v_{*} d v \\
\leq & \int_{|v|>1 / \sqrt{r}} f_{0}(v) \phi(v) d v+\frac{2 A_{0}}{\sqrt{r}} \int_{0}^{t}\|f(\tau)\|_{L_{1+\beta}^{1}}\|f(\tau)\|_{L_{\beta}^{1}} d \tau \\
\leq & \int_{|v|>1 / \sqrt{r}} f_{0}(v)\left(1+|v|^{2}\right) d v+\frac{2 A_{0}}{\sqrt{r}}\left\|f_{0}\right\|_{L_{2}^{1}}^{2} t \leq C \Phi_{f_{0}}(r), \quad t \in[0, r] .
\end{aligned}
$$

Therefore by identity $|f-g|=g-f+2(f-g)^{+}\left(\right.$here $\left.(x)^{+}=\max \{x, 0\}\right)$ we obtain (2.6):

$$
\begin{aligned}
U_{2}(t)= & \int_{\mathbb{R}^{3}}\left(g_{0}(v)-f_{0}(v)\right) \phi(v) d v+2 \int_{\mathbb{R}^{3}}(f(v, t)-g(v, t))^{+} \phi(v) d v \\
& \leq U_{2}(0)+\frac{4}{\sqrt{r}} U_{1}(t)+2 \int_{|v|>1 / \sqrt{r}} f(v, t) \phi(v) d v \\
& \leq U_{2}(0)+\frac{4}{\sqrt{r}} U_{1}(t)+C \Phi_{f_{0}}(r), \quad t \in[0, r] .
\end{aligned}
$$


Proof of (2.7): Denote

$$
\Pi_{\varepsilon}\left(f, f_{*}\right)=(1-\varepsilon f)\left(1-\varepsilon f_{*}\right) \quad \text { and } \quad d \mu=d \omega d v_{*} d v .
$$

It follows from the three inequalities

$$
\begin{array}{ll}
\left|f f_{*} \Pi_{\varepsilon}\left(f^{\prime}, f_{*}^{\prime}\right)-g g_{*} \Pi_{\varepsilon}\left(g^{\prime}, g_{*}^{\prime}\right)\right| \leq f\left|f_{*}-g_{*}\right|+g_{*}|f-g|+\varepsilon f f_{*}\left(\left|f^{\prime}-g^{\prime}\right|+\left|f_{*}^{\prime}-g_{*}^{\prime}\right|\right), & \quad \text { and } \\
\phi^{\prime} \leq \phi+\phi_{*} & \\
B \leq 2 b(\theta) \sqrt{\phi+\phi_{*}}, &
\end{array}
$$

and Lemma 3 with $k=0$ and $k=1$ that

$$
\begin{aligned}
U_{1}(t)-U_{1}(0) \leq & 2 \int_{0}^{t} d \tau \iiint_{\mathbb{R}^{3} \times \mathbb{R}^{3} \times S^{2}} B\left|f f_{*} \Pi_{\varepsilon}\left(f^{\prime}, f_{*}^{\prime}\right)-g g_{*} \Pi_{\varepsilon}\left(g^{\prime}, g_{*}^{\prime}\right)\right| \sqrt{\phi+\phi_{*}} d \mu \\
\leq & 4 A_{0} \int_{0}^{t} d \tau \iint_{\mathbb{R}^{3} \times \mathbb{R}^{3}}(f+g)\left|f_{*}-g_{*}\right|\left(\phi+\phi_{*}\right) d v_{*} d v \\
& +4 \int_{0}^{t} d \tau \int_{\mathbb{R}^{3}}|f-g| \sqrt{\phi}\left(\varepsilon \iint_{\mathbb{R}^{3} \times S^{2}} B f^{\prime} f_{*}^{\prime} d \omega d v_{*}\right) d v \\
& +4 \int_{0}^{t} d \tau \int_{\mathbb{R}^{3}}|f-g|\left(\varepsilon \iint_{\mathbb{R}^{3} \times S^{2}} B f^{\prime} f_{*}^{\prime} \sqrt{\phi_{*}} d \omega d v_{*}\right) d v \\
\leq & C \int_{0}^{t} U_{2}(\tau) d \tau, \quad t \geq 0 .
\end{aligned}
$$

This proves (2.7).

Proof of (2.8): We first prove an integrability result for the gain and the loss terms in the collision operator:

$$
\int_{r}^{t} d \tau \int_{\mathbb{R}^{3}} Q^{ \pm}(g)(v, \tau) \phi(v) \mathbb{1}_{\{f(v, \tau)>g(v, \tau)\}} d v<\infty, \quad t \geq r>0 .
$$

Using the integral representation of solution s to the Boltzmann equation (1.10), we find that

$$
\begin{aligned}
(f(v, t)-g(v, t))^{+}= & (f(v, r))-g(v, r))^{+} \\
& +\int_{r}^{t}(Q(f)(v, \tau)-Q(g)(v, \tau)) \mathbb{1}_{\{f(v, \tau)>g(v, \tau)\}} d \tau .
\end{aligned}
$$

This implies that for all $t>r>0$,

$$
\begin{aligned}
\int_{r}^{t} Q^{+}(g)(v, \tau) & \mathbb{1}_{\{f(v, \tau)>g(v, \tau)\}} d \tau \\
& \leq(f(v, r))-g(v, r))^{+}+\int_{r}^{t}\left(Q^{+}(f)(v, \tau)+Q^{-}(g)(v, \tau)\right) \mathbb{1}_{\{f(v, \tau)>g(v, \tau)\}} d \tau .
\end{aligned}
$$

Since $0 \leq 1-\varepsilon f, 1-\varepsilon g \leq 1$ and $\phi^{\prime}+\phi_{*}^{\prime}=\phi+\phi_{*}$, it follows that

$$
\begin{aligned}
\int_{r}^{t} d \tau \int_{\mathbb{R}^{3}}\left(Q^{+}(f)(v, \tau)\right. & \left.+Q^{-}(g)(v, \tau)\right) \mathbb{1}_{\{f(v, \tau)>g(v, \tau)\}} \phi(v) d v \\
& \leq \int_{r}^{t} d \tau \iiint_{\mathbb{R}^{3} \times \mathbb{R}^{3} \times S^{2}} B\left(f^{\prime} f_{*}^{\prime}+g g_{*}\right) \mathbb{1}_{\{f>g\}} \phi d \omega d v_{*} d v \\
& \leq \int_{r}^{t} d \tau \iiint_{\mathbb{R}^{3} \times \mathbb{R}^{3} \times S^{2}} B\left(f^{\prime} f_{*}^{\prime}+f g_{*}\right) \phi d \omega d v_{*} d v \\
& =\int_{r}^{t} d \tau \iiint_{\mathbb{R}^{3} \times \mathbb{R}^{3} \times S^{2}} B f\left(f_{*}+g_{*}\right) \phi d \omega d v_{*} d v \\
& \leq A_{0} \int_{r}^{t}\|f(\tau)\|_{L_{2+\beta}^{1}}\|f(\tau)+g(\tau)\|_{L_{\beta}^{1}} d \tau \leq C \int_{r}^{t}\left(1+\frac{1}{\tau}\right) d \tau<\infty
\end{aligned}
$$


In the last inequality, we have used (1.14). This proves (2.9). Together with (2.10), Lemma 1 and Lemma 3 (for $k=2$ ), we obtain

$$
\begin{aligned}
& U_{2}(t)-U_{2}(r)=\int_{r}^{t} d \tau \iiint_{\mathbb{R}^{3} \times \mathbb{R}^{3} \times S^{2}} B\left(f f_{*} \Pi_{\varepsilon}\left(f^{\prime}, f_{*}^{\prime}\right)-g g_{*} \Pi_{\varepsilon}\left(g^{\prime}, g_{*}^{\prime}\right)\right) \\
& \times\left(\phi^{\prime} \mathbb{1}_{\left\{f^{\prime}>g^{\prime}\right\}}+\phi_{*}^{\prime} \mathbb{1}_{\left\{f_{*}^{\prime}>g_{*}^{\prime}\right\}}-\phi \mathbb{1}_{\{f>g\}}-\phi_{*} \mathbb{1}_{\left\{f_{*}>g_{*}\right\}}\right) d \mu \\
& \leq 2 \int_{r}^{t} d \tau \iiint_{\mathbb{R}^{3} \times \mathbb{R}^{3} \times S^{2}} B f \phi\left|f_{*}-g_{*}\right| d \mu+2 \varepsilon \int_{r}^{t} d \tau \iiint_{\mathbb{R}^{3} \times \mathbb{R}^{3} \times S^{2}} B f f_{*}\left|f^{\prime}-g^{\prime}\right| \phi_{*}^{\prime} d \mu \\
& \leq 2 A_{0} \int_{r}^{t} d \tau \iint_{\mathbb{R}^{3} \times \mathbb{R}^{3}} f(\phi)^{(2+\beta) / 2}\left|f_{*}-g_{*}\right|\left(\phi_{*}\right)^{\beta / 2} d v_{*} d v \\
& +2 \int_{r}^{t} d \tau \int_{\mathbb{R}^{3}}|f-g|\left(\varepsilon \iint_{\mathbb{R}^{3} \times S^{2}} B f^{\prime} f_{*}^{\prime} \phi_{*} d \omega d v_{*}\right) d v \\
& \leq C \int_{r}^{t}\|f(\tau)\|_{L_{2+\beta}^{1}}\|f(\tau)-g(\tau)\|_{L_{1}^{1}} d \tau \leq C \int_{r}^{t}\left(1+\frac{1}{\tau}\right) U_{1}(\tau) d \tau, \quad t \geq r .
\end{aligned}
$$

This proves (2.8). As mentioned above, the three inequalities (2.6)-(2.8) imply the stability estimate (2.4).

Since the stability estimate (2.4) implies the uniqueness, the proof is complete once the existence of an energy conserving solution which satisfies the estimates (1.14) and (1.15).

But for $\varepsilon>0$, the Eq.(1.4) does have a conservation solution $f$ which satisfies the moment estimate (1.14)-(1.15). So the theorem holds for $\varepsilon>0$. For the classical case, the same estimates are implicit in e.g. [25] (see also [24]) and references there in, but to be complete, we give some details here. As usual, one constructs a sequence of solutions, $f_{n}$, which are obtained by a suitable truncation of initial data and of the equation. By inequality $\left|v-v_{*}\right|^{\beta} \geq\left(1+|v|^{2}\right)^{\beta / 2}-\left(1+\left|v_{*}\right|^{2}\right)^{\beta / 2}$ and the conservation of mass and energy, we have

$$
\begin{aligned}
\iint_{\mathbb{R}^{3} \times \mathbb{R}^{3}} f^{n}(v, t)\left(1+|v|^{2}\right)^{s / 2} & f^{n}\left(v_{*}, t\right)\left|v-v_{*}\right|^{\beta} d v_{*} d v \\
& \geq\left\|f_{0}^{n}\right\|_{L_{0}^{1}}\left\|f^{n}(t)\right\|_{L_{s+\beta}^{1}}-\left\|f_{0}^{n}\right\|_{L_{2}^{1}}\left\|f^{n}(t)\right\|_{L_{s}^{1}} .
\end{aligned}
$$

This can now be used to prove that there are constants $C_{n, s}$ such that

$$
\left\|f^{n}(t)\right\|_{L_{s}^{1}} \leq C_{n, s}\left(1+t^{-(s-2) / \beta}\right), \quad t>0, \quad s>2 .
$$

What is essential is that the constants $C_{n, s}$ depend only on initial data, and actually only on the mass and energy; moreover the constants are continuous as functions of $\left\|f_{0}^{n}\right\|_{2}^{1}$ and $\left\|f_{0}^{n}\right\|_{2}^{1}$.

Applying the estimate (2.4) to the solutions $f^{n}, f^{m}$ of the truncated Eq.(1.1), we have

$$
\sup _{t \geq 0} e^{-c_{n} t}\left\|f^{n}(t)-f^{m}(t)\right\|_{L_{2}^{1}} \leq C_{n} \Phi_{f_{0}}\left(\left\|f_{0}^{n}-f_{0}^{m}\right\|_{L_{2}^{1}},\right)
$$

where the coefficients $c_{n}$ and $C_{n}$ are uniformly bounded in $n$ (because of their continuous dependence of the norms of the initial data). It follows that if the sequence of initial data is a Cauchy sequence in $L_{2}^{1}$, then so is the sequence $f_{n}(\cdot, t)$, for all $t$. The limiting function $f$ is a solution to the Boltzmann equation, and by applying Fatou's lemma, (2.12) holds also for $f$.

Our next result is about the strong stability (continuity) with respective to the parameter $\varepsilon \in[0, \infty)$. The most interesting case is the continuity at $\varepsilon=0$ which in particular shows that the classical Boltzmann equation Eq.(1.1) is a strong limit of the quantum Boltzmann equation Eq.(1.4). A weak stability result of this kind has been obtained by Dolbeault [12]; his result is not restricted to the spatially homogeneous case.

Theorem 2. Let the kernel $B(z, \omega)$ be given by (1.8)-(1.4) with $0 \leq \beta \leq 1$. Let $f_{0}, f_{0}^{\varepsilon} \in L_{2}^{1}\left(\mathbb{R}^{3}\right)$ satisfying $f_{0} \geq 0,0 \leq f_{0}^{\varepsilon} \leq 1 / \varepsilon$. Let $f, f^{\varepsilon}$ be conservative solutions of Eq.(1.1) and Eq.(1.4) respectively with initial data $\left.f\right|_{t=0}=f_{0},\left.f^{\varepsilon}\right|_{t=0}=f_{0}^{\varepsilon}$. Suppose that $\left\|f_{0}^{\varepsilon}-f_{0}\right\|_{L_{2}^{1}} \rightarrow 0$ as $\varepsilon \rightarrow 0$. Then

$$
\sup _{t \geq 0} e^{-c t}\left\|f^{\varepsilon}(t)-f(t)\right\|_{L_{2}^{1}} \rightarrow 0 \quad(\varepsilon \rightarrow 0),
$$

where the constant $0<c<\infty$ depends only on $\left\|f_{0}\right\|_{L_{0}^{1}},\left\|f_{0}\right\|_{L_{2}^{1}}, \beta$, and on the angular function $b(\cdot)$. 
Proof: Let

$$
U_{\kappa}^{\varepsilon}(t)=\left\|f^{\varepsilon}(t)-f(t)\right\|_{L_{\kappa}^{1}}, \quad 0 \leq \kappa \leq 2 .
$$

We shall prove the following estimate, which implies (2.13):

$$
\sup _{t \geq 0} e^{-c t} U_{2}^{\varepsilon}(t) \leq C \Phi_{f_{0}}(\Lambda(\varepsilon)), \quad \forall 0<\varepsilon \leq \varepsilon_{0}
$$

where

$$
\begin{aligned}
& \Lambda(\varepsilon):=U_{2}^{\varepsilon}(0)+\varepsilon^{\frac{1}{6}}+A\left(\varepsilon^{\frac{1}{6}}\right)+\int_{0}^{\infty} \frac{1}{\sqrt{t}\left(1+t^{2}\right)}\left\|f(t) \mathbb{1}_{\left\{f(t) \phi>\varepsilon^{\left.-\frac{1}{6}\right\}}\right.}\right\|_{L_{2}^{1}} d t, \\
& A(\delta):=4 \pi\left(\int_{0}^{\delta} b(\theta) \sin (\theta) d \theta+\int_{\pi / 2-\delta}^{\pi / 2} b(\theta) \sin (\theta) d \theta\right), \quad 0<\delta \leq \pi / 4 .
\end{aligned}
$$

As before $\phi(v)=1+|v|^{2}$, and the constant $0<\varepsilon_{0} \leq\left(\frac{\pi}{4}\right)^{6}$ is to be taken so small that

$$
\Lambda(\varepsilon)<1 \quad \forall \varepsilon \in\left(0, \varepsilon_{0}\right] .
$$

The constants $0<c, C<\infty$ depend only on $\left\|f_{0}\right\|_{L_{0}^{1}},\left\|f_{0}\right\|_{L_{2}^{1}}, \beta$, and on the angular function $b(\cdot)$. It is easily seen that $\Lambda(\varepsilon) \rightarrow 0$ as $\varepsilon \rightarrow 0$.

We first prove (2.14) for $0<\beta \leq 1$. In this case, the solution $f$ satisfies the moment estimate (1.14)(1.15). Let $\Pi_{\varepsilon}\left(g, g_{*}\right)=\left(1-\varepsilon g^{\prime}\right)\left(1-\varepsilon g_{*}\right), d \mu=d \omega d v_{*} d v$, and

$$
g=f^{\varepsilon}, \quad g_{0}=f_{0}^{\varepsilon}
$$

and denote by $c, C$ the (different) constants mentioned above. Suppose $\varepsilon \in\left(0, \varepsilon_{0}\right]$. Our proof consists of several steps:

Step 1: Prove that for all $r \in[\Lambda(\varepsilon), 1]$

$$
U_{2}^{\varepsilon}(t) \leq C \Phi_{f_{0}}(r)+\frac{4}{\sqrt{r}} U_{1}^{\varepsilon}(t), \quad t \geq 0 .
$$

This can be proven in the same way as (2.6) provided one notices that $U_{2}^{\varepsilon}(0) \leq \Lambda(\varepsilon) \leq r \leq \Phi_{f_{0}}(r)$, etc.

Step 2: Prove that

$$
\begin{aligned}
U_{1}^{\varepsilon}(t) \leq & U_{1}^{\varepsilon}(0)+C \int_{0}^{t} U_{2}^{\varepsilon}(\tau) d \tau \\
& +C \int_{0}^{t}\left\|f(\tau) \mathbb{1}_{\left\{f(\tau) \phi>\varepsilon^{-\frac{1}{6}}\right\}}\right\|_{L_{2}^{1}} d \tau+C\left[\varepsilon^{\frac{1}{6}}+A\left(\varepsilon^{\frac{1}{6}}\right)\right] t, \quad t \geq 0 .
\end{aligned}
$$

Because $\phi, \phi^{\prime} \leq \phi \phi_{*}$, and because of the inequality $\left|f^{\prime} f_{*}^{\prime}-g^{\prime} g_{*}^{\prime} \Pi_{\varepsilon}\left(g, g_{*}\right)\right| \leq\left|f^{\prime} f_{*}^{\prime}-g^{\prime} g_{*}^{\prime}\right| \Pi_{\varepsilon}\left(g, g_{*}\right)+$ $f^{\prime} f_{*}^{\prime}\left(1-\Pi_{\varepsilon}\left(g, g_{*}\right)\right)$, and a similar inequality in the "un-primed" variables, it follows that

$$
\begin{aligned}
U_{1}^{\varepsilon}(t)- & U_{1}^{\varepsilon}(0) \leq \\
\leq & \int_{0}^{t} d \tau \iiint_{\mathbb{R}^{3} \times \mathbb{R}^{3} \times S^{2}} B\left|\left(f^{\prime} f_{*}^{\prime}-g^{\prime} g_{*}^{\prime} \Pi_{\varepsilon}\left(g, g_{*}\right)\right)-\left(f f_{*}-g g_{*} \Pi_{\varepsilon}\left(g^{\prime}, g_{*}^{\prime}\right)\right)\right| \sqrt{\phi} d \mu \\
\leq & 2 \int_{0}^{t} d \tau \iiint_{\mathbb{R}^{3} \times \mathbb{R}^{3} \times S^{2}} B\left|f f_{*}-g g_{*}\right| \sqrt{\phi \phi_{*}} d \mu \\
& +2 \int_{0}^{t} d \tau \iiint_{\mathbb{R}^{3} \times \mathbb{R}^{3} \times S^{2}} B f f_{*}\left[1-\Pi_{\varepsilon}\left(g^{\prime}, g_{*}^{\prime}\right)\right] \sqrt{\phi \phi_{*}} d \mu .
\end{aligned}
$$


Using $\left|v-v_{*}\right|^{\beta} \leq \sqrt{\phi(v) \phi\left(v_{*}\right)}$ one can estimate the first of the terms in the right hand side by

$$
\begin{array}{rl}
\int_{0}^{t} d \tau \iiint_{\mathbb{R}^{3} \times \mathbb{R}^{3} \times S^{2}} & B\left\{f\left|f_{*}-g_{*}\right|+g_{*}|f-g|\right\} \sqrt{\phi \phi_{*}} d \mu \\
\leq & A_{0} \int_{0}^{t} d \tau \iint_{\mathbb{R}^{3} \times \mathbb{R}^{3}}(f+g)\left|f_{*}-g_{*}\right| \phi \phi_{*} d v_{*} d v \\
\leq C \int_{0}^{t} U_{2}^{\varepsilon}(\tau) d \tau .
\end{array}
$$

In the second term in the right-hand side of (2.17), we let $\delta=\varepsilon^{\frac{1}{6}}$ and $R=\varepsilon^{-\frac{1}{6}}$; as before, $\theta=$ $\arccos \left(\left|\left\langle v-v_{*}, \omega\right\rangle\right| /\left|v-v_{*}\right|\right)$. We look separately at three parts of the domain of integration:

$$
\begin{aligned}
& \Omega_{1}=\left\{f_{*} \sqrt{\phi_{*}}>R\right\}, \\
& \Omega_{2}=\left\{f_{*} \sqrt{\phi_{*}} \leq R\right\} \cap(\{0 \leq \theta \leq \delta\} \cup\{\pi / 2-\delta \leq \theta \leq \pi / 2\}) \quad \text { and } \\
& \Omega_{3}=\left\{f_{*} \sqrt{\phi_{*}} \leq R\right\} \cap\{\delta \leq \theta \leq \pi / 2-\delta\} .
\end{aligned}
$$

Integration over the set $\Omega_{1}$, using the fact that $\int_{S^{2}} B \sqrt{\phi \phi_{*}} d \omega \leq \phi \phi_{*}$ gives a term bounded by

$$
A_{0}\left\|f_{0}\right\|_{L_{2}^{1}} \int_{0}^{t}\left\|f(\tau) \mathbb{1}_{\{f(\tau) \phi>R\}}\right\|_{L_{2}^{1}} d \tau .
$$

For the set $\Omega_{2}$, we similarly get a bound of the form

$$
A(\delta)\left\|f_{0}\right\|_{L_{2}^{1}} t \quad \text { where } \quad A(\delta)=C \int_{0}^{\delta} b(\theta) \sin (\theta) d \theta .
$$

Finally, for the third part, involving the domain $\Omega_{3}$, Lemma 2 can be used to obtain a bound of the form

$$
\begin{aligned}
& \varepsilon R 4 \pi \int_{\delta}^{\pi / 2-\delta}\left\{\frac{b(\theta) \sin (\theta)}{(\cos (\theta))^{3+\beta}}+\frac{b(\theta) \sin (\theta)}{(\sin (\theta))^{3+\beta}}\right\} d \theta \cdot \int_{0}^{t} \iint_{\mathbb{R}^{3} \times \mathbb{R}^{3}} f \sqrt{\phi} g_{*}\left|v-v_{*}\right|^{\beta} d v_{*} d v d \tau \\
& \quad \leq C \varepsilon R \delta^{-4}\left\|f_{0}\right\|_{L_{2}^{1}}\left\|g_{0}\right\|_{L_{2}^{1}} t .
\end{aligned}
$$

The terms (2.18), (2.19) and (2.20) together add up to the upper bound

$$
C \int_{0}^{t}\left\|f(\tau) \mathbb{1}_{\{f(\tau) \phi>R\}}\right\|_{L_{2}^{1}} d \tau+C\left[A\left(\varepsilon^{\frac{1}{6}}\right)+\varepsilon^{\frac{1}{6}}\right] t, \quad t \geq 0,
$$

which completes the proof of (2.16).

Step 3: Prove that

$$
\begin{aligned}
U_{2}^{\varepsilon}(t) \leq & U_{2}^{\varepsilon}(r)+C \int_{r}^{t}\left(1+\frac{1}{\tau}\right) U_{1}^{\varepsilon}(\tau) d \tau \\
& +C \int_{r}^{t}\left(1+\frac{1}{\tau}\right)\left\|f(\tau) \mathbb{1}_{\{f(\tau)>R\}}\right\|_{L_{1}^{1}} d \tau+C\left(\varepsilon^{\frac{1}{6}}+A\left(\varepsilon^{\frac{1}{6}}\right)\right) \int_{r}^{t}\left(1+\frac{1}{\tau}\right) d \tau, \quad t \geq r .
\end{aligned}
$$

The calculation is similar to that of Step 2. We have

$$
\begin{aligned}
& U_{2}^{\varepsilon}(t)-U_{2}^{\varepsilon}(r) \\
& =2 \int_{r}^{t} d \tau \iiint_{\mathbb{R}^{3} \times \mathbb{R}^{3} \times S^{2}} B\left(\left(f^{\prime} f_{*}^{\prime}-g^{\prime} g_{*}^{\prime} \Pi_{\varepsilon}\left(g, g_{*}\right)\right)-\left(f f_{*}-g g_{*} \Pi_{\varepsilon}\left(g^{\prime}, g_{*}^{\prime}\right)\right)\right) \phi \mathbb{1}_{\{f>g\}} d \mu \\
& \quad \leq 2 \int_{r}^{t} d \tau \iiint_{\mathbb{R}^{3} \times \mathbb{R}^{3} \times S^{2}} B\left(\left(f^{\prime} f_{*}^{\prime}-g^{\prime} g_{*}^{\prime}\right) \Pi_{\varepsilon}\left(g, g_{*}\right)-\left(f f_{*}-g g_{*}\right) \Pi_{\varepsilon}\left(g^{\prime}, g_{*}^{\prime}\right)\right) \phi \mathbb{1}_{\{f>g\}} d \mu \\
& \quad+2 \int_{r}^{t} d \tau \iiint_{\mathbb{R}^{3} \times \mathbb{R}^{3} \times S^{2}} B f^{\prime} f_{*}^{\prime}\left(1-\Pi_{\varepsilon}\left(g, g_{*}\right)\right) \phi d \mu, \quad t \geq r .
\end{aligned}
$$


For the first term, the identity $\phi^{\prime}+\phi_{*}^{\prime}=\phi+\phi_{*}$ and the inequality $\left(f f_{*}-g g_{*}\right)^{+}-\left(f f_{*}-g g_{*}\right) \mathbb{1}_{\{f>g\}} \leq$ $f\left|f_{*}-g_{*}\right|$ can be used together with the moment estimates (1.14)-(1.15) to get

$$
\begin{aligned}
2 \int_{r}^{t} d \tau & \iiint_{\mathbb{R}^{3} \times \mathbb{R}^{3} \times S^{2}} B\left(\left(f f_{*}-g g_{*}\right)^{+}-\left(f f_{*}-g g_{*}\right) \mathbb{1}_{\{f>g\}}\right) \Pi_{\varepsilon}\left(g^{\prime}, g_{*}^{\prime}\right) \phi d \mu \\
& \leq 2 \int_{r}^{t} d \tau \iiint_{\mathbb{R}^{3} \times \mathbb{R}^{3} \times S^{2}} B f \phi\left|f_{*}-g_{*}\right| d \mu \\
& \leq 2 A_{0} \int_{r}^{t}\|f(\tau)\|_{L_{\mathfrak{H}_{2+\beta}}} U_{1}^{\varepsilon}(\tau) d \tau \leq C \int_{r}^{t}\left(1+\frac{1}{\tau}\right) U_{1}^{\varepsilon}(\tau) d \tau, \quad t \geq r .
\end{aligned}
$$

To conclude the proof of (2.21), one can proceed as in Step 2 to see that the second term in (2.22) is smaller than

$$
C \int_{r}^{t}\left(1+\frac{1}{\tau}\right)\left\|f(\tau) \mathbb{1}_{\{f(\tau)>R\}}\right\|_{L_{1}^{1}} d \tau+C\left(\varepsilon^{\frac{1}{6}}+A\left(\varepsilon^{\frac{1}{6}}\right)\right) \int_{r}^{t}\left(1+\frac{1}{\tau}\right) d \tau, \quad t \geq r .
$$

Step 4: This concerns the interval $t \in[0,1]$, and the purpose is to prove that

$$
U_{2}^{\varepsilon}(t) \leq C \Phi_{f_{0}}(\Lambda(\varepsilon)), \quad t \in[0,1] .
$$

Let $r=\Lambda(\varepsilon)$. Then the estimates (2.16) and 2.21) together show that, for $r \leq t \leq 1$,

$$
\begin{aligned}
U_{2}^{\varepsilon}(t) \leq U_{2}^{\varepsilon}(r) & +C U_{1}^{\varepsilon}(0)|\log r| \\
& +C \int_{r}^{t} \frac{1}{\tau}\left(\int_{0}^{\tau} U_{2}^{\varepsilon}(\sigma) d \sigma+\int_{0}^{\tau}\left\|f(\sigma) \mathbb{1}_{\{f(\sigma) \phi>R\}}\right\|_{L_{2}^{1}} d \sigma\right) d \tau \\
& +C \int_{r}^{t} \frac{1}{\tau}\left(\varepsilon^{\frac{1}{6}}+A\left(\varepsilon^{\frac{1}{6}}\right)\right) \tau d \tau \\
& +C \int_{r}^{t} \frac{1}{\tau}\left\|f(\tau) \mathbb{1}_{\{f(\tau)>R\}}\right\|_{L_{1}^{1}} d \tau+C\left(\varepsilon^{\frac{1}{6}}+A\left(\varepsilon^{\frac{1}{6}}\right)\right)|\log r| .
\end{aligned}
$$

Let

$$
\lambda(\varepsilon)=\int_{0}^{\infty} \frac{1}{\sqrt{t}\left(1+t^{2}\right)}\left\|f(t) \mathbb{1}_{\{f(t) \phi>R\}}\right\|_{L_{2}^{1}} d t, \quad R=\varepsilon^{-\frac{1}{6}} .
$$

By the Fubini theorem, we have

$$
\begin{gathered}
\int_{r}^{t} \frac{1}{\tau}\left(\int_{0}^{\tau} U_{2}^{\varepsilon}(\sigma) d \sigma+\int_{0}^{\tau}\left\|f(\sigma) \mathbb{1}_{\{f(\sigma) \phi>R\}}\right\|_{L_{2}^{1}} d \sigma\right) d \tau \\
\quad \leq \int_{0}^{t}\left(U_{2}^{\varepsilon}(\sigma)+\left\|f(\sigma) \mathbb{1}_{\{f(\sigma) \phi>R\}}\right\|_{L_{2}^{1}}\right)|\log \sigma| d \sigma \\
\quad \leq \int_{0}^{t} U_{2}^{\varepsilon}(\sigma)|\log \sigma| d \sigma+C \lambda(\varepsilon), \quad t \in[r, 1]
\end{gathered}
$$

and

$$
\int_{r}^{t} \frac{1}{\tau}\left\|f(\tau) \mathbb{1}_{\{f(\tau)>R\}}\right\|_{L_{1}^{1}} d \tau \leq C \frac{1}{\sqrt{r}} \lambda(\varepsilon), \quad t \in[r, 1] .
$$

Since $\lambda(\varepsilon) \leq \Lambda(\varepsilon)=r$ and $|\log r| \leq \frac{1}{\sqrt{r}}$, it follows from definition of $\Lambda(\varepsilon)$ that

$$
U_{2}^{\varepsilon}(t) \leq U_{2}^{\varepsilon}(r)+C \sqrt{r}+C \int_{0}^{t} U_{2}^{\varepsilon}(\tau)|\log \tau| d \tau, \quad t \in[r, 1] .
$$

On the other hand, combining (2.15) with (2.16), gives

$$
U_{2}^{\varepsilon}(t) \leq C \Phi_{f_{0}}(r)=C \Phi_{f_{0}}(\Lambda(\varepsilon)), \quad t \in[0, r] .
$$

Thus

$$
U_{2}^{\varepsilon}(t) \leq C \Phi_{f_{0}}(\Lambda(\varepsilon))+C \int_{0}^{t} U_{2}^{\varepsilon}(\tau)|\log \tau| d \tau, \quad t \in[0,1] .
$$

and the Gronwall lemma implies (2.23). 
Step 5: Estimate on $[0, \infty)$ :

$$
U_{2}^{\varepsilon}(t) \leq C \Phi_{f_{0}}(\Lambda(\varepsilon)) e^{c t}, \quad t \in[0, \infty) .
$$

We first prove this estimate on $[1, \infty)$ : Taking $r=1$ in $(2.21)$ and using the Gronwall lemma together with $U_{2}^{\varepsilon}(1) \leq C \Phi_{f_{0}}(\Lambda(\varepsilon))$ ( this comes from (2.23) ) and the inequality $t^{\alpha} \leq e^{\alpha t}(\alpha>0)$, we obtain

$$
\begin{aligned}
U_{2}^{\varepsilon}(t) & \leq\left(U_{2}^{\varepsilon}(1)+C \int_{1}^{t}\left\|f(\tau) \mathbb{1}_{\{f(\tau)>R\}}\right\|_{L_{2}^{1}} d \tau+C\left(^{\left(\frac{1}{6}\right.}+A\left(\varepsilon^{\frac{1}{6}}\right)\right),\right) e^{c(t-1)} \\
& \leq C\left\{U_{2}^{\varepsilon}(1)+\Lambda(\varepsilon)\right\} e^{c t} \leq C \Phi_{f_{0}}(\Lambda(\varepsilon)) e^{c t}, \quad t \in[1, \infty) .
\end{aligned}
$$

This together with Step 4 (2.23) implies (2.24), and the proof of theorem is completed.

The remaining part of this section is devoted to the proofs of Lemma 1 and of 3; Lemma 2.

Proof of Lemma 1: The calculations carried out here are fully independent of the value of $\varepsilon$, and hence we set $\varepsilon=1$. We then prove (2.1) by separately checking the following eight different cases, which together cover all possibilities:
(1) $f^{\prime}>g^{\prime}, f_{*}^{\prime}>g_{*}^{\prime}$;
(5) $\quad f^{\prime}>g^{\prime}, f_{*}^{\prime} \leq g_{*}^{\prime}, f>g, f_{*} \leq g_{*}$;
(2) $f^{\prime} \leq g^{\prime}, f_{*}^{\prime} \leq g_{*}^{\prime}$
(6) $f^{\prime} \leq g^{\prime}, f_{*}^{\prime}>g_{*}^{\prime}, f>g, f_{*} \leq g_{*}$;
(3) $f>g, f_{*}>g_{*}$;
(7) $f^{\prime}>g^{\prime}, f_{*}^{\prime} \leq g_{*}^{\prime}, f \leq g, f_{*}>g_{*}$;
(4) $f \leq g, f_{*} \leq g_{*}$;
(8) $f^{\prime} \leq g^{\prime}, f_{*}^{\prime}>g_{*}^{\prime}, f \leq g, f_{*}>g_{*}$.

The proof for cases (1)-(4) and (5)-(8) have many points in common. Here we only give the case (1) and case (5). Let $\Delta(f, g ; \phi)$ be the left-hand side of the above inequality:

$$
\begin{aligned}
\Delta(f, g ; \phi)= & \left(\left(1-f^{\prime}\right)\left(1-f_{*}^{\prime}\right)-g g_{*}\left(1-g^{\prime}\right)\left(1-g_{*}^{\prime}\right)\right) \\
& \times\left({ }^{\prime} \mathbb{1}_{\left\{f^{\prime}>g^{\prime}\right\}}+\phi_{*}^{\prime} \mathbb{1}_{\left\{f_{*}^{\prime}>g_{*}^{\prime}\right\}}-\phi \mathbb{1}_{\{f>g\}}-\phi_{*} \mathbb{1}_{\left\{f_{*}>g_{*}\right\}}\right) .
\end{aligned}
$$

Case (1): Here $f^{\prime}>g^{\prime}$ and $f_{*}^{\prime}>g_{*}^{\prime}$. Because $\phi^{\prime}+\phi_{*}^{\prime}=\phi+\phi_{*}$, we can deduce that

$$
\phi^{\prime} \mathbb{1}_{\left\{f^{\prime}>g^{\prime}\right\}}+\phi_{*}^{\prime} \mathbb{1}_{\left\{f_{*}^{\prime}>g_{*}^{\prime}\right\}}-\phi \mathbb{1}_{\{f>g\}}+\phi_{*} \mathbb{1}_{\left\{f_{*}>g_{*}\right\}}=\phi \mathbb{1}_{\{f \leq g\}}+\phi_{*} \mathbb{1}_{\left\{f_{*} \leq g_{*}\right\}} .
$$

Again because $0 \leq 1-f^{\prime} \leq 1-g^{\prime}, 0 \leq 1-f_{*}^{\prime} \leq 1-g_{*}^{\prime}$, and because $f$ and $g$ are non-negative,

$$
\begin{aligned}
\Delta(f, g, ; \phi) & =\left(f f_{*}\left(1-f^{\prime}\right)\left(1-f_{*}^{\prime}\right)-g g_{*}\left(1-g^{\prime}\right)\left(1-g_{*}^{\prime}\right)\right)\left(\phi \mathbb{1}_{\{f \leq g\}}+\phi_{*} \mathbb{1}_{\left\{f_{*} \leq g_{*}\right\}}\right) \\
& \leq\left(f f_{*}-g g_{*}\right)\left(1-g^{\prime}\right)\left(1-g_{*}^{\prime}\right)\left(\phi \mathbb{1}_{\{f \leq g\}}+\phi_{*} \mathbb{1}_{\left\{f_{*} \leq g_{*}\right\}}\right) \\
& =\left(f f_{*}-g g_{*}\right)\left(1-g^{\prime}\right)\left(1-g_{*}^{\prime}\right) \phi \mathbb{1}_{\{f \leq g\}}+\left(f f_{*}-g g_{*}\right)\left(1-g^{\prime}\right)\left(1-g_{*}^{\prime}\right) \phi_{*} \mathbb{1}_{\left\{f_{*} \leq g_{*}\right\}} \\
& \leq f\left(f_{*}-g_{*}\right)\left(1-g^{\prime}\right)\left(1-g_{*}^{\prime}\right) \phi \mathbb{1}_{\{f \leq g\}}+f_{*}(f-g)\left(1-g^{\prime}\right)\left(1-g_{*}^{\prime}\right) \phi_{*} \mathbb{1}_{\left\{f_{*} \leq g_{*}\right\}} \\
& \leq f\left|f_{*}-g_{*}\right| \phi+f_{*}|f-g| \phi_{*}=(f \phi)\left|f_{*}-g_{*}\right|+(f \phi)_{*}|f-g|,
\end{aligned}
$$

which concludes this case.

Case (5): Here $f^{\prime}>g^{\prime}, f_{*}^{\prime} \leq g_{*}^{\prime}, f>g$ and $f_{*} \leq g_{*}$. We have

$$
\phi^{\prime} \mathbb{1}_{\left\{f^{\prime}>g^{\prime}\right\}}+\phi_{*}^{\prime} \mathbb{1}_{\left\{f_{*}^{\prime}>g_{*}^{\prime}\right\}}-\phi \mathbb{1}_{\{f>g\}}+\phi_{*} \mathbb{1}_{\left\{f_{*}>g_{*}\right\}}=\phi^{\prime}-\phi
$$

and

$$
\Delta(f, g, ; \phi)=\left(f f_{*}\left(1-f^{\prime}\right)\left(1-f_{*}^{\prime}\right)-g g_{*}\left(1-g^{\prime}\right)\left(1-g_{*}^{\prime}\right)\right)\left(\phi^{\prime}-\phi\right)
$$


Here it is convenient to treat separately the cases $\phi^{\prime}>\phi$ and $\phi^{\prime} \leq \phi$. In the first case,

$$
\begin{aligned}
\Delta(f, g, ; \phi)= & f f_{*}\left(\left(1-f^{\prime}\right)\left(1-f_{*}^{\prime}\right)-\left(1-g^{\prime}\right)\left(1-g_{*}^{\prime}\right)\right)\left(\phi^{\prime}-\phi\right) \\
& +\left(f f_{*}-g g_{*}\right)\left(1-g^{\prime}\right)\left(1-g_{*}^{\prime}\right)\left(\phi^{\prime}-\phi\right) \\
\leq & \left.f f_{*}\left(1-g^{\prime}\right)\left(\left(1-f_{*}^{\prime}\right)-\left(1-g_{*}^{\prime}\right)\right)\left(\phi^{\prime}-\phi\right) \quad \text { (because } 0 \leq 1-f^{\prime} \leq 1-g^{\prime}\right) \\
& +(f-g) f_{*}\left(1-g^{\prime}\right)\left(1-g_{*}^{\prime}\right)\left(\phi^{\prime}-\phi\right) \quad\left(\text { because } g g_{*} \geq g f_{*}\right) \\
\leq & f f_{*} f_{*}^{\prime}-g_{*}^{\prime}\left|\left(\phi^{\prime}-\phi\right)+\right| f-g \mid f_{*}\left(\phi^{\prime}-\phi\right) \quad\left(\text { because } \phi^{\prime}-\phi \leq \phi_{*}\right) \\
\leq & f f_{*}\left|f_{*}^{\prime}-g_{*}^{\prime}\right| \phi^{\prime}+|f-g| f_{*} \phi_{*} \quad(f \phi)_{*}|f-g|+f f_{*}\left|f_{*}^{\prime}-g_{*}^{\prime}\right| \phi^{\prime} .
\end{aligned}
$$

On the other hand, when $\phi^{\prime}-\phi \leq 0$, we have

$$
\begin{aligned}
\Delta(f, g, ; \phi)= & \left(g g_{*}\left(1-g^{\prime}\right)\left(1-g_{*}^{\prime}\right)-f f_{*}\left(1-f^{\prime}\right)\left(1-f_{*}^{\prime}\right)\right)\left(\phi-\phi^{\prime}\right) \\
= & \left(g g_{*}-f f_{*}\right)\left(1-g^{\prime}\right)\left(1-g_{*}^{\prime}\right)\left(\phi-\phi^{\prime}\right)+f f_{*}\left(\left(1-g^{\prime}\right)\left(1-g_{*}^{\prime}\right)-\left(1-f^{\prime}\right)\left(1-f_{*}^{\prime}\right)\right)\left(\phi-\phi^{\prime}\right) \\
\leq & f\left(g_{*}-f_{*}\right)\left(1-g^{\prime}\right)\left(1-g_{*}^{\prime}\right)\left(\phi-\phi^{\prime}\right) \quad(\text { because } g<f) \\
& +f f_{*}\left(1-f_{*}^{\prime}\right)\left(\left(1-g^{\prime}\right)-\left(1-f^{\prime}\right)\right)\left(\phi-\phi^{\prime}\right) \quad\left(\text { because } 0 \leq 1-g_{*}^{\prime} \leq 1-f_{*}^{\prime}\right) \\
\leq & f\left|g_{*}-f_{*}\right| \phi+f f_{*}\left|g^{\prime}-f^{\prime}\right|\left(\phi_{*}^{\prime}-\phi_{*}\right) \quad\left(\text { because } 0 \leq \phi-\phi^{\prime}=\phi_{*}^{\prime}-\phi_{*} \leq \phi_{*}^{\prime}\right) \\
\leq & (f \phi)\left|f_{*}-g_{*}\right|+f f_{*}\left|f^{\prime}-g^{\prime}\right| \phi_{*}^{\prime} .
\end{aligned}
$$

In a similar way analysing the cases (2) - (4) and (6) - (8) gives the desired estimate for all cases.

Proof of Lemma 2: We need to estimate $\varepsilon \int_{\mathbb{R}^{3} \times S^{2}} B\left(v-v_{*}, \omega\right) f^{\prime} f_{*}^{\prime}\left(1+\left|v_{*}\right|^{2}\right)^{k / 2} d \omega d v_{*}$. We can assume that $0<k<3$; in fact, the case $k=0$ is contained in the proof for $k>0$, and the case $k=3$ can proven by taking the limit $k \rightarrow 3$. It is clear that the integral is bounded by $2^{k / 2}(I(v)+J(v))$, where

$$
\begin{aligned}
& I(v)=\varepsilon \iint_{\mathbb{R}^{3} \times S^{2}} B\left(v-v_{*}, \omega\right) f^{\prime} f_{*}^{\prime} d \omega d v_{*}, \\
& J(v)=\varepsilon \iint_{\mathbb{R}^{3} \times S^{2}} B\left(v-v_{*}, \omega\right) f^{\prime} f_{*}^{\prime}\left|v_{*}\right|^{k} d \omega d v_{*} .
\end{aligned}
$$

Let $B=B_{1}+B_{2}$, where $B_{1}\left(v-v_{*}, \omega\right)=b(\theta) \mathbb{1}_{[0, \pi / 4]}(\theta)\left|v-v_{*}\right|^{\beta}$ and (on the complementary set) $B_{2}\left(v-v_{*}, \omega\right)=b(\theta)\left|v-v_{*}\right|^{\beta} \mathbb{1}_{(\pi / 4, \pi / 2]}(\theta)$. Similarly, $b_{1}(\theta)=b(\theta) \mathbb{1}_{[0, \pi / 4]}(\theta)$, and $b_{2}(\theta)=b(\theta)-b_{1}(\theta)$. By assumption, $0 \leq f \leq 1 / \varepsilon$ and then using Lemma 2 , we can deduce

$$
\begin{aligned}
I(v) & \leq \iint_{\mathbb{R}^{3} \times S^{2}} B_{1}\left(v-v_{*}, \omega\right) f\left(v^{\prime}\right) d \omega d v_{*}+\iint_{\mathbb{R}^{3} \times S^{2}} B_{2}\left(v-v_{*}, \omega\right) f\left(v_{*}^{\prime}\right) d \omega d v_{*} \\
& =4 \pi\left\{\int_{0}^{\pi / 2} \frac{b_{1}(\theta) \sin (\theta)}{(\cos (\theta))^{3+\beta}} d \theta+\int_{0}^{\pi / 2} \frac{b_{2}(\theta) \sin (\theta)}{(\sin (\theta))^{3+\beta}} d \theta\right\} \int_{\mathbb{R}^{3}} f\left(v_{*}\right)\left|v-v_{*}\right|^{\beta} d v_{*} \\
& =4 \pi\left\{\int_{0}^{\pi / 4} \frac{b(\theta) \sin (\theta)}{(\cos (\theta))^{3+\beta}} d \theta+\int_{\pi / 4}^{\pi / 2} \frac{b(\theta) \sin (\theta)}{(\sin (\theta))^{3+\beta}} d \theta\right\} \int_{\mathbb{R}^{3}} f\left(v_{*}\right)\left|v-v_{*}\right|^{\beta} d v_{*},
\end{aligned}
$$

and finally see that $I(v) \leq 2^{2} A_{0}\|f\|_{L_{\beta}^{1}}\left(1+|v|^{2}\right)^{\beta / 2}$.

Next split $J(v)=J_{1}(v)+J_{2}(v)+J_{3}(v)+J_{4}(v)$, where

$$
\begin{aligned}
& J_{1}(v)=\varepsilon \iint_{\mathbb{R}^{3} \times S^{2}} B_{1}\left(v-v_{*}, \omega\right) f^{\prime} f_{*}^{\prime}\left|v_{*}\right|^{k} 1_{\left\{\left|v_{*}\right| \leq 2\left|v^{\prime}\right|\right\}} d \omega d v_{*}, \\
& J_{2}(v)=\varepsilon \iint_{\mathbb{R}^{3} \times S^{2}} B_{2}\left(v-v_{*}, \omega\right) f^{\prime} f_{*}^{\prime}\left|v_{*}\right|^{k} 1_{\left\{\left|v_{*}\right| \leq 2\left|v_{*}^{\prime}\right|\right\}} d \omega d v_{*}, \\
& J_{3}(v)=\varepsilon \iint_{\mathbb{R}^{3} \times S^{2}} B_{1}\left(v-v_{*}, \omega\right) f^{\prime} f_{*}^{\prime}\left|v_{*}\right|^{k} 1_{\left\{\left|v_{*}\right|>2\left|v^{\prime}\right|\right\}} d \omega d v_{*}, \\
& J_{4}(v)=\varepsilon \iint_{\mathbb{R}^{3} \times S^{2}} B_{2}\left(v-v_{*}, \omega\right) f^{\prime} f_{*}^{\prime}\left|v_{*}\right|^{k} 1_{\left\{\left|v_{*}\right|>2\left|v_{*}^{\prime}\right|\right\}} d \omega d v_{*} .
\end{aligned}
$$


For $J_{1}(v)$ and $J_{2}(v)$ we can again use Lemma 2 to find the inequalities

$$
\begin{aligned}
& J_{1}(v)+J_{2}(v) \\
& \leq 2^{k} \iint_{\mathbb{R}^{3} \times S^{2}}\left\{B_{1}\left(v-v_{*}, \omega\right) f\left(v^{\prime}\right)\left|v^{\prime}\right|^{k}+B_{2}(v-v *, \omega) f\left(v_{*}^{\prime}\right)\left|v_{*}^{\prime}\right|^{k}\right\} d \omega d v_{*} \\
& =2^{k} \cdot 4 \pi\left\{\int_{0}^{\pi / 4} \frac{b(\theta) \sin (\theta)}{(\cos (\theta))^{3+\beta}} d \theta+\int_{\pi / 4}^{\pi / 2} \frac{b(\theta) \sin (\theta)}{(\sin (\theta))^{3+\beta}} d \theta\right\} \int_{\mathbb{R}^{3}} f\left(v_{*}\right)\left|v_{*}\right|^{k}\left|v-v_{*}\right|^{\beta} d v_{*} \\
& \leq 2^{5} A_{0}\|f\|_{L_{k+\beta}^{1}}\left(1+|v|^{2}\right)^{\beta / 2} .
\end{aligned}
$$

Next, to estimate $J_{3}(v)$ and $J_{4}(v)$, we first note that

$$
\begin{aligned}
& \left|v_{*}\right|>2\left|v^{\prime}\right| \text { implies }\left|v_{*}\right| \leq 2\left|v-v_{*}\right| \sin (\theta) \\
& \left|v_{*}\right|>2\left|v_{*}^{\prime}\right| \text { implies }\left|v_{*}\right| \leq 2\left|v-v_{*}\right| \cos (\theta)
\end{aligned}
$$

Beginning with $J_{3}(v)$ (the calculations for $J_{4}(v)$ are essentially the same), we split this term once more:

$$
\begin{aligned}
J_{3}(v) & \leq 2^{k} \varepsilon \iint_{\mathbb{R}^{3} \times S^{2}} B_{1}\left(v-v_{*}, \omega\right) f^{\prime} f_{*}^{\prime}\left|v-v_{*}\right|^{k} \sin ^{k}(\theta) d \omega d v_{*} \\
& =2^{k} \varepsilon \iint_{\mathbb{R}^{3} \times S^{2}} b_{1}(\theta) \sin ^{k}(\theta) f^{\prime} f_{*}^{\prime}\left|v-v_{*}\right|^{k+\beta} d \omega d v_{*} \\
& =J_{31}(v)+J_{32}(v),
\end{aligned}
$$

where

$$
\begin{aligned}
& J_{31}(v)=2^{k} \varepsilon \iint_{\mathbb{R}^{3} \times S^{2}} b_{1}(\theta) \sin ^{k}(\theta) f^{\prime} f_{*}^{\prime}\left|v-v_{*}\right|^{k+\beta} \mathbb{1}_{\left\{\left|v_{*}^{\prime}\right| \leq\left|v^{\prime}\right|\right\}} d \omega d v_{*}, \quad \text { and } \\
& J_{32}(v)=2^{k} \varepsilon \iint_{\mathbb{R}^{3} \times S^{2}} b_{1}(\theta) \sin ^{k}(\theta) f^{\prime} f_{*}^{\prime}\left|v-v_{*}\right|^{k+\beta} \mathbb{1}_{\left\{\left|v_{*}^{\prime}\right|>\left|v^{\prime}\right|\right\}} d \omega d v_{*} .
\end{aligned}
$$

Because $\left|v-v_{*}\right|=\left|v^{\prime}-v_{*}^{\prime}\right|$, Lemma 2 can again and this gives

$$
\begin{aligned}
J_{31}(v) & \leq 2^{k} \cdot 2^{k+\beta} \iint_{\mathbb{R}^{3} \times S^{2}} b_{1}(\theta) \sin ^{k}(\theta) f\left(v^{\prime}\right)\left|v^{\prime}\right|^{k+\beta} d \omega d v_{*} \\
& =2^{2 k+\beta} \cdot 4 \pi \int_{0}^{\pi / 4} \frac{b(\theta) \sin ^{k+1}(\theta)}{\cos ^{3}(\theta)} d \theta \int_{\mathbb{R}^{3}} f\left(v_{*}\right)\left|v_{*}\right|^{k+\beta} d v_{*} \\
& \leq 2^{9} A_{0}\|f\|_{L_{k+\beta}^{1}},
\end{aligned}
$$

and for $J_{32}(v)$, finally, we use Hölder's inequality which leads to the estimate

$$
\begin{aligned}
J_{32}(v) \leq & 2^{k} \varepsilon\left(\iint_{\mathbb{R}^{3} \times S^{2}} b_{1}(\theta)\left|v-v_{*}\right|^{k+\beta}\left(f\left(v^{\prime}\right)\right)^{p} d \omega d v_{*}\right)^{1 / p} \\
& \times\left(\iint_{\mathbb{R}^{3} \times S^{2}} b_{1}(\theta) \sin ^{k q}(\theta)\left|v-v_{*}\right|^{k+\beta}\left(f\left(v_{*}^{\prime}\right)\right)^{q} \mathbb{1}_{\left\{\left|v_{*}^{\prime}\right|>\left|v^{\prime}\right|\right\}} d \omega d v_{*}\right)^{1 / q} .
\end{aligned}
$$

In the integrals, we replace $\varepsilon$ by $1 / f$, which shows that $J_{32}(v)$ is bounded by

$$
\begin{aligned}
2^{k}\left(\iint_{\mathbb{R}^{3} \times S^{2}} b_{1} *(\theta)\left|v-v_{*}\right|^{k+\beta} f\left(v^{\prime}\right) d \omega d v_{*}\right)^{1 / p} & \\
& \times\left(2^{k+\beta} \iint_{\mathbb{R}^{3} \times S^{2}} b_{1}(\theta) \sin ^{k q}(\theta) f\left(v_{*}^{\prime}\right)\left|v_{*}^{\prime}\right|^{k+\beta} d \omega d v_{*}\right)^{1 / q} .
\end{aligned}
$$


By choosing $\frac{1}{p}=1-\frac{k}{3}$ and $\frac{1}{q}=\frac{k}{3}$, and once more referring to Lemma 2 gives the following bound for $J_{32}(v)$ :

$$
\begin{aligned}
2^{k}\left(4 \pi \int_{0}^{\pi / 4} \frac{b(\theta) \sin (\theta)}{\cos ^{3+k+\beta}(\theta)} d \theta \int_{\mathbb{R}^{3}} f\left(v_{*}\right)\left|v-v_{*}\right|^{k+\beta} d v_{*}\right)^{(3-k) / 3} \\
\quad \times\left(2^{k+\beta} 4 \pi \int_{0}^{\pi / 4} \frac{b(\theta) \sin (\theta)}{\sin ^{3}(\theta)} \sin ^{3}(\theta) d \theta \int_{\mathbb{R}^{3}} f\left(v_{*}\right)\left|v_{*}\right|^{k+\beta} d v_{*}\right)^{k / 3} \\
\leq 2^{k} \cdot\left(2^{(3+k+\beta) / 2} A_{0}\|f\|_{L_{k+\beta}^{1}}\left(1+|v|^{2}\right)^{(k+\beta) / 2}\right)^{(3-k) / 3}\left(2^{k+\beta} A_{0}\|f\|_{L_{k+\beta}^{1}}\right)^{k / 3} \\
\leq 2^{7} A_{0}\|f\|_{L_{k+\beta}^{1}}\left(1+|v|^{2}\right)^{\alpha / 2} .
\end{aligned}
$$

This gives

$$
J_{3}(v) \leq 2^{10} A_{0}\|f\|_{L_{k+\beta}^{1}}\left(1+|v|^{2}\right)^{\alpha / 2}
$$

and in the same way

$$
J_{4}(v) \leq 2^{10} A_{0}\|f\|_{L_{k+\beta}^{1}}\left(1+|v|^{2}\right)^{\alpha / 2}
$$

Combining the estimates of the different terms gives the estimate in the lemma.

\section{Strong Convergence to Equilibrium}

The main result of this section, is that the solutions to the spatially homogeneous Boltzmann equation for Fermi-Dirac particles converge strongly to equilibrium, at least under the "high temperature" condition mentioned in the introduction. The weak convergence in $L^{1}$ has already been established in [20]. As for the classical Boltzmann equation, the strong convergence is related to regularity properties of the gain term; it is close to being strongly compact in $L^{1}$.

We begin by stating the main theorem, and then, before entering into the details of the proof, we state and prove some lemmas that are the main ingredients of the proof.

Theorem 3. Suppose the collision kernel $B(z, \omega)$ is given by (1.8)-(1.9). Let $f_{0} \in L_{2}^{1}\left(\mathbb{R}^{3}\right)$ satisfy $0 \leq$ $f_{0} \leq 1 / \varepsilon$ on $\mathbb{R}^{3}$, and $M_{0}:=\left\|f_{0}\right\|_{L^{1}}>0$. Let $T, T_{F}$ be the corresponding temperature and the Fermi temperature respectively, and suppose that $T>\frac{2}{5} T_{F}$. Let $f$ be the unique conservative solution of Eq. (1.4) with $\left.f\right|_{t=0}=f_{0}$, and let $F=F_{a, b}$ be the Fermi-Dirac distribution having the same mass, mean velocity and temperature. Then

$$
\|f(t)-F\|_{L^{1}}^{2} \leq 2\left\|f_{0}\right\|_{L^{1}}[S(F)-S(f(t))] \leq C\|f(t)-F\|_{L_{2}^{1}}, \quad t \geq 0,
$$

where $C<\infty$ depends only on $\left\|f_{0}\right\|_{L^{1}},\left\|f_{0}\right\|_{L_{2}^{1}}$ and $\varepsilon$. Furthermore, if $\beta>0$ and $T \geq \gamma(1) T_{F}$, then

$$
\|f(t)-F\|_{L_{2}^{1}} \rightarrow 0 \quad(t \rightarrow \infty)
$$

where $\gamma(x)$ is the function defi ned in (1.13). If $\beta=0$ and $f_{0} \in L_{s}^{1}\left(\mathbb{R}^{3}\right)$ for some $s>2$, then with same condition $T \geq \gamma(1) T_{F}$, the strong convergence (3.2) still holds.

For this section, one can change from one value of $\varepsilon$ to another, simply by rescaling the equation, and hence it is convenient to set $\varepsilon=1$, and in the same way there is no restriction in assuming that $\int_{\mathbb{R}^{3}} f_{0}(v) v d v=0$.

The first inequality in (3.1) has been established in [20]; it is really a kind of Csiszár-Kullback inequality. The second inequality follows by an elementary inequality,

$$
\begin{aligned}
& (1-y) \log (1-y)+y \log y-(1-x) \log (1-x)-x \log x \\
& \leq(y-x) \log \left(\frac{x}{1-x}\right)+|y-x|\left(1+\log \left(\frac{1}{x}\right)+\frac{1}{1-x}\right), \quad(0<x<1,0 \leq y \leq 1)
\end{aligned}
$$


which can be proven by using

$$
\begin{aligned}
&(1-y) \log (1-y)+ y \log y-(1-x) \log (1-x)-x \log x \\
&=(y-x) \log \left(\frac{x}{1-x}\right)+(1-y) \log \left(\frac{1-y}{1-x}\right)+y \log \left(\frac{y}{x}\right) \quad \text { and } \\
& y \log \left(\frac{y}{x}\right)=(y-x) \log \left(\frac{y}{x}\right)+x \log \left(\frac{y}{x}\right) \leq(y-x) \log \left(\frac{1}{x}\right)+y-x, \quad(y>x) .
\end{aligned}
$$

Then (3.1) follows by choosing $x=F(v)=a e^{-b|v|^{2}} /\left(1+a e^{-b|v|^{2}}\right)$, and $y=f(v, t)$, and then using the conservation properties of the solutions.

The proof of (3.2) is much more complicated, and relies a number of auxiliary results. The most important of these is a version of Lions' compactness result for the gain term of the collision operator (see P.L. Lions [18], but also Lu [23], or [8] and [30] for related results).

Let $\mathbb{C}$ be the set of all complex numbers. For any $s \in \mathbf{R}$, let $L_{s}^{1}\left(\mathbb{R}^{3} \times \mathbb{R}^{3}\right)$ be the weighted $L^{1}$-space as introduced before, i.e.

$L_{s}^{1}\left(\mathbb{R}^{3} \times \mathbb{R}^{3}\right):=\left\{\Psi: \mathbb{R}^{3} \times \mathbb{R}^{3} \mapsto \mathbb{C} \mid \Psi\right.$ is measurable on $\mathbb{R}^{3} \times \mathbb{R}^{3}$ and

$$
\left.\|\Psi\|_{L_{s}^{1}\left(\mathbb{R}^{3} \times \mathbb{R}^{3}\right)}=\iint_{\mathbb{R}^{3} \times \mathbb{R}^{3}}\left|\Psi\left(v, v_{*}\right)\right|\left(1+|v|^{2}+\left|v_{*}\right|^{2}\right)^{s / 2} d v<\infty\right\} .
$$

The gain term (and in the same way, the loss term) in the collision operator, corresponds in a natural way to linear operator $\mathcal{Q}^{+}: L_{s}^{1}\left(\mathbb{R}^{3} \times \mathbb{R}^{3}\right) \rightarrow L_{s^{\prime}}^{1}\left(\mathbb{R}^{3}\right)$, and in the Fermi-Dirac case it is also natural to consider a modified operator $\mathcal{Q}^{+}(\Psi \mid F)$ :

$$
\begin{aligned}
& \mathcal{Q}^{+}(\Psi)(v)=\iint_{\mathbb{R}^{3} \times S^{2}} B\left(v-v_{*}, \omega\right) \Psi\left(v^{\prime}, v_{*}^{\prime}\right) d \omega d v_{*}, \\
& \mathcal{Q}^{+}(\Psi \mid F)(v)=\iint_{\mathbb{R}^{3} \times S^{2}} B\left(v-v_{*}, \omega\right) \Psi\left(v^{\prime}, v_{*}^{\prime}\right) F\left(v_{*}\right) d \omega d v_{*} .
\end{aligned}
$$

For our main result, $F$ will be chosen a Fermi-Dirac distribution (1.2).

Lemma 4. Let $B(z, \omega)$ be given by (1.8)-(1.9) with $0 \leq \beta \leq 1$. Let $\mathcal{K}$ be a subset of $L_{2 \beta}^{1}\left(\mathbb{R}^{3} \times \mathbb{R}^{3}\right) \cap$ $L^{2}\left(\mathbb{R}^{3} \times \mathbb{R}^{3}\right)$ satisfying the following conditions:

$$
\begin{aligned}
& \sup _{\Psi \in \mathcal{K}}\left\{\|\Psi\|_{L_{\beta}^{1, \infty}}+\|\Psi\|_{L_{2 \beta}^{1}}+\|\Psi\|_{L^{2}}\right\}<\infty, \\
& \sup _{\Psi \in \mathcal{K}} \iint_{|v|+\left|v_{*}\right|>R}\left|\Psi\left(v, v_{*}\right)\right|\left(1+|v|^{2}+\left|v_{*}\right|^{2}\right)^{\beta} d v d v_{*} \rightarrow 0(R \rightarrow \infty) .
\end{aligned}
$$

Here

$$
\|\Psi\|_{L_{\beta}^{1, \infty}}:=\int_{\mathbb{R}^{3}}\left(\|\Psi(v, \cdot)\|_{L^{\infty}}+\|\Psi(\cdot, v)\|_{L^{\infty}}\right)\left(1+|v|^{2}\right)^{\beta / 2} d v .
$$

Let $F$ be any given smooth function such that $F \in L^{1}\left(\mathbb{R}^{3}\right) \cap L^{\infty}\left(\mathbb{R}^{3}\right)$ and $\hat{F} \in L^{1}\left(\mathbb{R}^{3}\right)$. $(\hat{F}(\xi)=$ $\int_{\mathbb{R}^{3}} F\left(v_{*}\right) e^{-\mathrm{i}\left\langle\xi, v_{*}\right\rangle} d v_{*}$ denotes the Fourier transform of $F$.) Then

- the sets $\left\{\mathcal{Q}^{+}(\Psi)\right\}_{\Psi \in \mathcal{K}}$ and $\left\{\mathcal{Q}^{+}(\Psi \mid F)\right\}_{\Psi \in \mathcal{K}}$ are both relatively compact in $L^{2}\left(\mathbb{R}^{3}\right)$, and

- the operators $\mathcal{Q}^{+}(\cdot)$ and $\mathcal{Q}^{+}(\cdot \mid F)$ are continuous in the sense that for any sequence $\left\{\Psi_{n}\right\}_{n \geq 1} \subset$ $\mathcal{K}$, which is converging weakly to 0 in $L^{1}\left(\mathbb{R}^{3} \times \mathbb{R}^{3}\right)$,

$$
\lim _{n \rightarrow \infty}\left\|\mathcal{Q}^{+}\left(\Psi_{n}\right)\right\|_{L^{2}}=0 \text { and } \lim _{n \rightarrow \infty}\left\|\mathcal{Q}^{+}\left(\Psi_{n} \mid F\right)\right\|_{L^{2}}=0
$$

This way of treating the gain term as a linear operator acting in both $v$ and $v_{*}$ was used independently in [23] and in [8]. To prove Lemma 4, we first prove 5:

Lemma 5. Let $B(z, \omega)$ and $\beta$ be given as in Lemma 4 , and let $\Psi$ be measurable on $\mathbb{R}^{3} \times \mathbb{R}^{3}$ and satisfy $\|\Psi\|_{L_{\beta}^{1, \infty}}<\infty$ where $\|\Psi\|_{L_{\beta}^{1, \infty}}$ is defi ned in (3.6). Then

$$
\left|\mathcal{Q}^{+}(\Psi)(v)\right| \leq 8 A_{0}\|\Psi\|_{L_{\beta}^{1, \infty}}\left(1+|v|^{2}\right)^{\beta / 2} \quad \text { a.e. } v \in \mathbb{R}^{3} .
$$


Proof: Let $\psi_{1}(v)=\|\Psi(v, \cdot)\|_{L^{\infty}}, \psi_{2}(v)=\|\Psi(\cdot, v)\|_{L^{\infty}}$. Then by the Fubini theorem, $\left|\Psi\left(v, v_{*}\right)\right| \leq$ $\min \left\{\psi_{1}(v), \psi_{2}\left(v_{*}\right)\right\}$ a.e. $\left(v, v_{*}\right) \in \mathbb{R}^{3} \times \mathbb{R}^{3}$. Therefore by applying Lemma 2 , (or more directly by Lemma 2 in ref. [22]), for all $\varphi \in L^{1}\left(\mathbb{R}^{3}\right)$,

$$
\left\|\mathcal{Q}^{+}(\Psi) \varphi\right\|_{L_{-\beta}^{1}} \leq 8 A_{0}\left(\left\|\psi_{1}\right\|_{L_{\beta}^{1}}+\left\|\psi_{2}\right\|_{L_{\beta}^{1}}\right)\|\varphi\|_{L^{1}}=8 A_{0}\|\Psi\|_{L_{\beta}^{1, \infty}}\|\varphi\|_{L^{1}}
$$

This implies (3.7).

Proof of Lemma 4: We first extend the set $\mathcal{K}$ to a larger one, which still is denoted $\mathcal{K}$, with the property that if $|\chi|=1$ and $\Psi \in \mathcal{K}$ then $\chi \Psi \in \mathcal{K}$, where $\chi$ is any complex valued measurable function on $\mathbb{R}^{3} \times \mathbb{R}^{3}$. It is obvious that this extension does not change the $L^{\infty}$-bounds in (3.4)-(3.5). It is easy to check that the sets $\left\{\mathcal{Q}^{+}(\Psi)\right\}_{\Psi \in \mathcal{K}}$ and $\left\{\mathcal{Q}^{+}(\Psi \mid F)\right\}_{\Psi \in \mathcal{K}}$ are bounded both in $L_{\beta}^{1}\left(\mathbb{R}^{3}\right)$ and $L^{2}\left(\mathbb{R}^{3}\right)$ (compare also with estimates below; there one can set $R=0)$ :

$$
\begin{aligned}
\left\|\mathcal{Q}^{+}(\Psi)\right\|_{L_{\beta}^{1}} & \leq 2 A_{0}\|\Psi\|_{L_{2 \beta}^{1}}, \\
\left\|\mathcal{Q}^{+}(\Psi \mid F)\right\|_{L_{\beta}^{1}} & \leq 2 A_{0}\|F\|_{L^{\infty}}\|\Psi\|_{L_{2 \beta}^{1}}, \\
\left\|\mathcal{Q}^{+}(\Psi)\right\|_{L^{2}}^{2} & \leq 16 A_{0}\|\Psi\|_{L_{\beta}^{1, \infty}}\|\Psi\|_{L_{2 \beta}^{1}}, \\
\left\|\mathcal{Q}^{+}(\Psi \mid F)\right\|_{L^{2}}^{2} & \leq 16 A_{0}\|F\|_{L^{\infty}}^{2}\|\Psi\|_{L_{\beta}^{1, \infty}}^{1,}\|\Psi\|_{L_{2 \beta}^{1}} .
\end{aligned}
$$

Now we prove the $L^{2}$-compactness of $\left\{\mathcal{Q}^{+}(\Psi)\right\}_{\Psi \in \mathcal{K}}$ and $\left\{\mathcal{Q}^{+}(\Psi \mid F)\right\}_{\Psi \in \mathcal{K}}$. For any $R \geq 0$, Lemma 5 gives

$$
\int_{|v|>R}\left|\mathcal{Q}^{+}(\Psi)(v)\right|^{2} d v \leq C \int_{\mathbb{R}^{3}}\left|\mathcal{Q}^{+}(\Psi)(v)\right|\left(1+|v|^{2}\right)^{\beta / 2} 1_{\{|v|>R\}} d v,
$$

where $C=8 A_{0} \sup _{\Psi \in \mathcal{K}}\|\Psi\|_{L_{\beta}^{1, \infty}}<\infty$, and (3.8) is in turn bounded by

$$
\begin{aligned}
& C \iiint_{\mathbb{R}^{3} \times \mathbb{R}^{3} \times S^{2}} B\left(v-v_{*}, \omega\right)\left|\Psi\left(v, v_{*}\right)\right|\left(1+\left|v^{\prime}\right|^{2}\right)^{\beta / 2} 1_{\left\{\left|v^{\prime}\right|>R\right\}} d \omega d v_{*} d v \\
& \quad \leq C \iiint_{\mathbb{R}^{3} \times \mathbb{R}^{3} \times S^{2}} B\left(v-v_{*}, \omega\right)\left|\Psi\left(v, v_{*}\right)\right|\left(1+|v|^{2}+\left|v_{*}\right|^{2}\right)^{\beta / 2} 1_{\left\{|v|+\left|v_{*}\right|>R\right\}} d \omega d v_{*} d v \\
& \quad \leq 2 C \iint_{|v|+\left|v_{*}\right|>R}\left|\Psi\left(v, v_{*}\right)\right|\left(1+|v|^{2}+\left|v_{*}\right|^{2}\right)^{\beta} d \omega d v_{*} d v .
\end{aligned}
$$

Thus by the condition (3.5) we have

$$
\sup _{\Psi \in \mathcal{K}} \int_{|v|>R}\left|\mathcal{Q}^{+}(\Psi)(v)\right|^{2} d v \rightarrow 0 \quad(R \rightarrow \infty) .
$$

Next we prove that

$$
\sup _{\Psi \in \mathcal{K}}\left\|\mathcal{Q}^{+}(\Psi)(\cdot+h)-\mathcal{Q}^{+}(\Psi)\right\|_{L^{2}} \rightarrow 0 \quad(h \rightarrow 0) .
$$

Let

$$
B_{R}(z, \omega)=B(z, \omega) 1_{\{|z| \leq R\}}=b(\theta)|z|^{\beta} 1_{\{|z| \leq R\}}
$$

and let $\mathcal{Q}_{R}^{+}(\cdot)$ be the gain operator corresponding to the smaller kernel $B_{R}(z, \omega)$. Since $\int_{S^{2}} B_{R}(\cdot, \omega) d \omega \in$ $L^{2}\left(\mathbb{R}^{3}\right)$, it follows from the regularity property of Boltzmann gain operator $\mathcal{Q}^{+}(\cdot)$ that there is a positive measurable function $\xi \mapsto K_{R}(|\xi|)$, which is determined only by the kernel $B_{R}(\cdot, \cdot)$, such that

$$
\int_{\mathbb{R}^{3}} K_{R}(|\xi|)\left|\mathcal{Q}_{R}^{+}(\Psi)^{\wedge}(\xi)\right|^{2} d \xi \leq\|\Psi\|_{L^{2}}^{2}
$$

and

$$
K_{R}(|\xi|) \rightarrow \infty \quad \text { as }|\xi| \rightarrow \infty
$$


This is essentially Lions' compactness result, (see [8], [18], [23] and [23]). Now (3.11) and (3.12) together with the identity

$$
\left\|\mathcal{Q}_{R}^{+}(\Psi)(\cdot+h)-\mathcal{Q}_{R}^{+}(\Psi)\right\|_{L^{2}}^{2}=(2 \pi)^{-3} \int_{\mathbb{R}^{3}}\left|\mathcal{Q}_{R}^{+}(\Psi)^{\wedge}(\xi)\right|^{2}\left|e^{\mathrm{i}\langle\xi, h\rangle}-1\right|^{2} d \xi
$$

and

$$
\sup _{\Psi \in \mathcal{K}, \xi \in \mathbb{R}^{3}}\left|\mathcal{Q}_{R}^{+}(\Psi)^{\wedge}(\xi)\right| \leq \sup _{\Psi \in \mathcal{K}}\left\|\mathcal{Q}^{+}(\Psi)\right\|_{L^{1}}<\infty
$$

imply

$$
\sup _{\Psi \in \mathcal{K}}\left\|\mathcal{Q}_{R}^{+}(\Psi)(\cdot+h)-\mathcal{Q}_{R}^{+}(\Psi)\right\|_{L^{2}} \rightarrow 0 \quad(h \rightarrow 0) \quad \forall R>0 .
$$

On the other hand, because $\left|v^{\prime}-v_{*}^{\prime}\right|=\left|v-v_{*}\right|$, we have $\mathcal{Q}^{+}(\Psi)-\mathcal{Q}_{R}^{+}(\Psi)=\mathcal{Q}^{+}\left(\Psi_{R}\right)$, where $\Psi_{R}\left(v, v_{*}\right)=$ $\mathbb{1}_{\left\{\left|v-v_{*}\right|>R\right\}} \Psi\left(v, v_{*}\right)$. Thus by the $L^{2}$-estimate and the condition (3.5),

$$
\begin{aligned}
& \sup _{\Psi \in \mathcal{K}}\left\|\mathcal{Q}^{+}(\Psi)-\mathcal{Q}_{R}^{+}(\Psi)\right\|_{L^{2}}^{2}=\sup _{\Psi \in \mathcal{K}}\left\|\mathcal{Q}^{+}\left(\Psi_{R}\right)\right\|_{L^{2}}^{2} \\
& \quad \leq 2 C \sup _{\Psi \in \mathcal{K}} \int_{|v|+\left|v_{*}\right|>R}\left|\Psi\left(v, v_{*}\right)\right|\left(1+|v|^{2}+\left|v_{*}\right|^{2}\right)^{\beta} d v d v_{*} \rightarrow 0 \quad(R \rightarrow \infty) .
\end{aligned}
$$

Thus the triangle inequality

$$
\left\|\mathcal{Q}^{+}(\Psi)(\cdot+h)-\mathcal{Q}^{+}(\Psi)\right\|_{L^{2}} \leq\left\|\mathcal{Q}_{R}^{+}(\Psi)(\cdot+h)-\mathcal{Q}_{R}^{+}(\Psi)\right\|_{L^{2}}+2\left\|\mathcal{Q}^{+}(\Psi)-\mathcal{Q}_{R}^{+}(\Psi)\right\|_{L^{2}}
$$

together with (3.14) and (3.15) implies (3.10) which together with (3.9) implies the compactness of $\left\{\mathcal{Q}^{+}(\Psi)\right\} \Psi \in \mathcal{K}$ in $L^{2}\left(\mathbb{R}^{3} \times \mathbb{R}^{3}\right)$.

To prove the $L^{2}$-compactness of $\left\{\mathcal{Q}^{+}(\Psi \mid F)\right\}_{\Psi \in \mathcal{K}}$, we consider the inverse Fourier transform of $F$ :

$$
F\left(v_{*}\right)=(2 \pi)^{-3} \int_{\mathbb{R}^{3}} \hat{F}(\xi) e^{\mathrm{i}\left\langle\xi, v_{*}\right\rangle} d \xi .
$$

By assumption $\hat{F} \in L^{1}\left(\mathbb{R}^{3}\right)$. Since $e^{\mathrm{i}\left\langle\xi, v_{*}\right\rangle}=e^{-\mathrm{i}\langle\xi, v\rangle} e^{\mathrm{i}\left\langle\xi, v^{\prime}\right\rangle} e^{\mathrm{i}\left\langle\xi, v_{*}^{\prime}\right\rangle}$, it follows by Fubini's theorem, that

$$
\mathcal{Q}^{+}(\Psi \mid F)(v)=(2 \pi)^{-3} \int_{\mathbb{R}^{3}} \hat{F}(\xi) e^{-\mathrm{i}\langle\xi, v\rangle} \mathcal{Q}^{+}\left(\chi_{\xi} \Psi\right)(v) d \xi
$$

where

$$
\chi_{\xi}\left(v, v_{*}\right)=e^{\mathrm{i}\langle\xi, v\rangle} e^{\mathrm{i}\left\langle\xi, v_{*}\right\rangle} .
$$

By definition, $\chi_{\xi} \Psi \in \mathcal{K}$, and so the Minkovski inequality can be applied to (3.16), and this gives

$$
\begin{aligned}
\left\|\mathcal{Q}^{+}(\Psi \mid F)(\cdot+h)-\mathcal{Q}^{+}(\Psi \mid F)\right\|_{L^{2}} \\
\leq(2 \pi)^{-3} \int_{\mathbb{R}^{3}}|\hat{F}(\xi)|\left\|\mathcal{Q}^{+}\left(\chi_{\xi} \Psi\right)(\cdot+h) e^{-\mathrm{i}\langle\xi, h\rangle}-\mathcal{Q}^{+}\left(\chi_{\xi} \Psi\right)\right\|_{L^{2}} d \xi \\
\leq(2 \pi)^{-3} \sup _{\Psi \in \mathcal{K}}\left\|\mathcal{Q}^{+}(\Psi)\right\|_{L^{2}} \int_{\mathbb{R}^{3}}|\hat{F}(\xi)|\left|e^{-i\langle\xi, h\rangle}-1\right| d \xi \\
\quad+(2 \pi)^{-3} \int_{\mathbb{R}^{3}}|\hat{F}(\xi)| d \xi \sup _{\Psi \in \mathcal{K}}\left\|\mathcal{Q}^{+}(\Psi)(\cdot+h)-\mathcal{Q}^{+}(\Psi)\right\|_{L^{2}} .
\end{aligned}
$$

This implies that

$$
\sup _{\Psi \in \mathcal{K}}\left\|\mathcal{Q}^{+}(\Psi \mid F)(\cdot+h)-\mathcal{Q}^{+}(\Psi \mid F)\right\|_{L^{2}} \rightarrow 0 \quad(h \rightarrow 0) .
$$

because of the assumed $L^{2}$-bounds on $\hat{F}$, and the already obtained estimate on $\left\|\mathcal{Q}^{+}(\Psi)(\cdot+h)-\mathcal{Q}^{+}(\Psi)\right\|_{L^{2}}$ (the inequality (3.10)). And equation (3.9) leads to the estimate

$$
\sup _{\Psi \in \mathcal{K}} \int_{|v|>R}\left|\mathcal{Q}^{+}(\Psi \mid F)(v)\right|^{2} d v \leq\|F\|_{L^{\infty}}^{2} \sup _{\Psi \in \mathcal{K}} \int_{|v|>R}\left|\mathcal{Q}^{+}(\Psi)(v)\right|^{2} d v \rightarrow 0(R \rightarrow \infty) .
$$

Thus the set $\left\{\mathcal{Q}^{+}\left(\Psi_{t} \mid F\right)\right\}_{\Psi \in \mathcal{K}}$ is also relatively compact in $L^{2}\left(\mathbb{R}^{3}\right)$. This proves the first part of Lemma 4 . 
To prove the second part of the Lemma, let $\Psi_{n}$ be a sequence in $\mathcal{K}$ that is weakly converging to zero in $L^{1}\left(\mathbb{R}^{3} \times \mathbb{R}^{3}\right)$. We have to prove that $\lim _{n \rightarrow \infty}\left\|\mathcal{Q}^{+}\left(\Psi_{n}\right)\right\|_{L^{2}}=0$ and that $\lim _{n \rightarrow \infty}\left\|\mathcal{Q}^{+}\left(\Psi_{n} \mid F\right)\right\|_{L^{2}}=0$. For any $R>0$ we have

$$
\begin{aligned}
& \mathcal{Q}_{R}^{+}\left(\Psi_{n}\right)^{\wedge}(\xi) \\
& \quad=\iint_{\mathbb{R}^{3} \times \mathbb{R}^{3}} \Psi_{n}\left(v, v_{*}\right)\left(\int_{S^{2}} B_{R}\left(v-v_{*}, \omega\right) e^{\mathrm{i}\left\langle\xi, v^{\prime}\right\rangle} d \omega\right) d v d v_{*} \rightarrow 0 \quad(n \rightarrow \infty) .
\end{aligned}
$$

Also (3.13) we can deduce $L^{\infty}$-bounds on $\sup _{n \geq 1} \sup _{\xi \in \mathbb{R}^{3}}\left|\mathcal{Q}_{R}^{+}\left(\Psi_{n}\right)^{\wedge}(\xi)\right|<\infty$. Combining this with (3.11), (3.12) and using the dominated convergence theorem gives

$$
\left\|\mathcal{Q}_{R}^{+}\left(\Psi_{n}\right)\right\|_{L^{2}}^{2}=(2 \pi)^{3} \int_{\mathbb{R}^{3}}\left|\mathcal{Q}_{R}^{+}\left(\Psi_{n}\right)^{\wedge}(\xi)\right|^{2} d \xi \rightarrow 0 \quad(n \rightarrow \infty) \quad \forall R>0 .
$$

Therefore in the inequality

$$
\left\|\mathcal{Q}^{+}\left(\Psi_{n}\right)\right\|_{L^{2}} \leq\left\|\mathcal{Q}_{R}^{+}\left(\Psi_{n}\right)\right\|_{L^{2}}+\sup _{\Psi \in \mathcal{K}}\left\|\mathcal{Q}^{+}(\Psi)-\mathcal{Q}_{R}^{+}(\Psi)\right\|_{L^{2}}
$$

first letting $n \rightarrow \infty$ then letting $R \rightarrow \infty$ (using (3.15)) we obtain that $\lim _{n \rightarrow \infty}\left\|\mathcal{Q}^{+}\left(\Psi_{n}\right)\right\|_{L^{2}}=0$. Next by (3.16) and the Minkovski inequality,

$$
\left\|\mathcal{Q}^{+}\left(\Psi_{n} \mid F\right)\right\|_{L^{2}} \leq(2 \pi)^{-3 / 2} \int_{\mathbb{R}^{3}}|\hat{F}(\xi)|\left\|\mathcal{Q}^{+}\left(\chi_{\xi} \Psi_{n}\right)\right\|_{L^{2}} d \xi
$$

It is obvious that for any $\xi \in \mathbb{R}^{3},\left\{\chi_{\xi} \Psi_{n}\right\}_{n \geq 1} \subset \mathcal{K}$ and that $\chi_{\xi} \Psi_{n} \rightarrow 0$ weakly in $L^{1}\left(\mathbb{R}^{3} \times \mathbb{R}^{3}\right)$. Thus $\lim _{n \rightarrow \infty}\left\|\mathcal{Q}^{+}\left(\chi_{\xi} \Psi_{n}\right)\right\|_{L^{2}}=0, \forall \xi \in \mathbb{R}^{3}$. Since

$$
\sup _{n \geq 1, \xi \in \mathbb{R}^{3}}\left\|\mathcal{Q}^{+}\left(\chi_{\xi} \Psi_{n}\right)\right\|_{L^{2}} \leq \sup _{\Psi \in \mathcal{K}}\left\|\mathcal{Q}^{+}(\Psi)\right\|_{L^{2}}<\infty
$$

and $\hat{F} \in L^{1}\left(\mathbb{R}^{3}\right)$, it follows from the dominated convergence theorem that the right hand side of (3.17) tends to 0 as $n \rightarrow \infty$. This completes the proof of Lemma 4 .

The next lemma is a general result about weak convergence of products. It is naturally used in the study of kinetic equations, and one version can be found for example in [6]. The proof is short, and we present it here for completeness.

Lemma 6. Let $\left\{P_{t}\right\}_{t \geq t_{0}},\left\{Q_{t}\right\}_{t \geq t_{0}}$ be two families of complex-valued measurable functions defi ned on $\mathbf{R}^{N}$ which satisfy one of the following two conditions $(*),(* *)$ :

$*\left\{P_{t}\right\}_{t \geq t_{0}}$ is relatively weakly compact in $L^{1}\left(\mathbf{R}^{N}\right), \sup _{t \geq t_{0}}\left\|Q_{t}\right\|_{L^{\infty}\left(\mathbf{R}^{N}\right)}<\infty$, and $\lim _{t \rightarrow \infty} Q_{t}(v)=0$ a.e. $v \in \mathbf{R}^{N}$;

** $\left\{P_{t}\right\}_{t \geq t_{0}},\left\{Q_{t}\right\}_{t \geq t_{0}} \subset L^{2}\left(\mathbf{R}^{N}\right), P_{t} \rightarrow 0(t \rightarrow \infty)$ weakly in $L^{2}\left(\mathbf{R}^{N}\right)$, and $\left\{Q_{t}\right\}_{t \geq t_{0}}$ is relatively compact in $L^{2}\left(\mathbf{R}^{N}\right)$.

Then

$$
\lim _{t \rightarrow \infty} \int_{\mathbf{R}^{N}} P_{t}(v) Q_{t}(v) d v=0 .
$$

Proof: The proof for the condition $(*)$ is easy: in fact in this case we have $\left\|P_{t} Q_{t}\right\|_{L^{1}} \rightarrow 0(t \rightarrow \infty)$. Now suppose the condition $(* *)$ is satisfied. Choose a sequence $t_{n} \rightarrow \infty(n \rightarrow \infty)$ such that

$$
\limsup _{t \rightarrow \infty}\left|\int_{\mathbf{R}^{N}} P_{t}(v) Q_{t}(v) d v\right|=\lim _{n \rightarrow \infty}\left|\int_{\mathbf{R}^{N}} P_{t_{n}}(v) Q_{t_{n}}(v) d v\right|=: A .
$$

By the compactness of $\left\{Q_{t}\right\}_{t \geq t_{0}}$, there exist a subsequence $\left\{t_{n_{k}}\right\}_{k=1}^{\infty}$ of $\left\{t_{n}\right\}_{n=1}^{\infty}$ and a function $Q_{\infty} \in$ $L^{2}\left(\mathbf{R}^{N}\right)$ such that $\left\|Q_{t_{n_{k}}}-Q_{\infty}\right\|_{L^{2}} \rightarrow 0(k \rightarrow \infty)$. Since $\left\{P_{t_{n}}\right\}_{n=1}^{\infty}$ is weakly convergent, it is bounded: $C=\sup _{n \geq 1}\left\|P_{t_{n}}\right\|_{L^{2}}<\infty$. Therefore, because $P_{t_{n}} \rightarrow 0(n \rightarrow \infty)$ weakly in $L^{2}$, we obtain

$$
\begin{aligned}
A & =\lim _{k \rightarrow \infty}\left|\int_{\mathbf{R}^{N}} P_{t_{n_{k}}}(v) Q_{t_{n_{k}}}(v) d v\right| \\
& \leq C \lim _{k \rightarrow \infty}\left\|Q_{t_{n_{k}}}-Q_{\infty}\right\|_{L^{2}}+\lim _{k \rightarrow \infty}\left|\int_{\mathbf{R}^{N}} P_{t_{n_{k}}}(v) Q_{\infty}(v) d v\right|=0,
\end{aligned}
$$


which ends the proof.

All that remains in this paper, is the Proof of Theorem 3. Actually, the proof of the first part is given just after the statement, and the full theorem is proven when it has been established that

$$
\|f(t)-F\|_{L^{2}} \rightarrow 0 \quad(t \rightarrow \infty) .
$$

In fact, using the identity $|f-F|=f-F+2(F-f)^{+}$we find that for any $R>0$ and for $C_{R}=$ $2\left(1+R^{2}\right)\left(\frac{4 \pi}{3} R^{3}\right)^{1 / 2}$,

$$
\|f(t)-F\|_{L_{2}^{1}} \leq C_{R}\|f(t)-F\|_{L^{2}}+2 \int_{|v|>R} F(v)\left(1+|v|^{2}\right) d v,
$$

which means that the convergence (3.19) implies (3.2).

To prove (3.19), we first prove that

$$
\begin{aligned}
& \frac{1}{2} \frac{d}{d t}\|f(t)-F\|_{L^{2}}^{2} \\
& \quad \leq-\int_{\mathbb{R}^{3}}(f-F)^{2}\left(\iint_{\mathbb{R}^{3} \times S^{2}} B F_{*}\left(1-F^{\prime}-F_{*}^{\prime}\right) d \omega d v_{*}\right) d v+W(t),
\end{aligned}
$$

for some function $W(t)$ satisfying $W(t) \rightarrow 0(t \rightarrow \infty)$. The proof is now carried out in several steps.

Step 1: The function $t \mapsto\|f(t)-F\|_{L^{2}}^{2}$ is in $C^{1}[0, \infty)$ and

$$
\frac{1}{2} \frac{d}{d t}\|f(t)-F\|_{L^{2}}^{2}=\int_{\mathbb{R}^{3}} Q(f)(v, t)(f(v, t)-F(v)) d v, \quad t \geq 0 .
$$

This is easy, however, the it requires some steps of calculation to verify that the right-hand side of (3.21) is continuous.

Step 2: Here we expand the right-hand side of (3.21). Using the identities

$$
\begin{aligned}
& f^{\prime} f_{*}^{\prime}(1-f)\left(1-f_{*}\right)-f f_{*}\left(1-f^{\prime}\right)\left(1-f_{*}^{\prime}\right)=f^{\prime} f_{*}^{\prime}\left(1-f-f_{*}\right)-f f_{*}\left(1-f^{\prime}-f_{*}^{\prime}\right), \\
& \quad \text { and } \\
& F^{\prime} F_{*}^{\prime}\left(1-F-F_{*}\right)-F F_{*}\left(1-F^{\prime}-F_{*}^{\prime}\right)=0
\end{aligned}
$$

we find that

$$
\begin{aligned}
\left(f^{\prime} f_{*}^{\prime}\right. & \left.(1-f)\left(1-f_{*}\right)-f f_{*}\left(1-f^{\prime}\right)\left(1-f_{*}^{\prime}\right)\right)(f-F) \\
= & \left(f^{\prime} f_{*}^{\prime}-F^{\prime} F_{*}^{\prime}\right)\left(1-F-F_{*}\right)(f-F)-f^{\prime} f_{*}^{\prime}\left(f+f_{*}-F-F_{*}\right)(f-F) \\
& -f\left(f_{*}-F_{*}\right)\left(1-F^{\prime}-F_{*}^{\prime}\right)(f-F)-F_{*}(f-F)^{2}\left(1-F^{\prime}-F_{*}^{\prime}\right) \\
& +f f_{*}\left(f^{\prime}+f_{*}^{\prime}-F^{\prime}-F_{*}^{\prime}\right)(f-F) .
\end{aligned}
$$

Then we can compute the integrals of each of the five terms in the right hand side separately. The first term becomes

$$
\begin{array}{rl}
\iiint_{\mathbb{R}^{3} \times \mathbb{R}^{3} \times S^{2}} & B\left(f^{\prime} f_{*}^{\prime}-F^{\prime} F_{*}^{\prime}\right)\left(1-F-F_{*}\right)(f-F) d \omega d v_{*} d v \\
= & \int_{\mathbb{R}^{3}}(1-F)(f-F)\left(\iint_{\mathbb{R}^{3} \times S^{2}} B\left(f^{\prime} f_{*}^{\prime}-F^{\prime} F_{*}^{\prime}\right) d \omega d v_{*}\right) d v \\
& -\int_{\mathbb{R}^{3}}(f-F)\left(\iint_{\mathbb{R}^{3} \times S^{2}} B\left(f^{\prime} f_{*}^{\prime}-F^{\prime} F_{*}^{\prime}\right) F_{*} d \omega d v_{*}\right) d v
\end{array}
$$

and the third and fourth are

$$
\begin{array}{rl}
\iiint_{\mathbb{R}^{3} \times \mathbb{R}^{3} \times S^{2}} & B\left(-f\left(f_{*}-F_{*}\right)\left(1-F^{\prime}-F_{*}^{\prime}\right)(f-F)\right) d \omega d v_{*} d v \\
= & -\int_{\mathbb{R}^{3}} f(f-F)\left(\int_{\mathbb{R}^{3}}\left(f_{*}-F_{*}\right)\left(\int_{S^{2}} B\left(1-F^{\prime}-F_{*}^{\prime}\right) d \omega\right) d v_{*}\right) d v
\end{array}
$$


and

$$
\begin{aligned}
\iiint_{\mathbb{R}^{3} \times \mathbb{R}^{3} \times S^{2}} B & \left(-F_{*}(f-F)^{2}\left(1-F^{\prime}-F_{*}^{\prime}\right)\right) d \omega d v_{*} d v \\
= & -\int_{\mathbb{R}^{3}}(f-F)^{2}\left(\iint_{\mathbb{R}^{3} \times S^{2}} B F_{*}\left(1-F^{\prime}-F_{*}^{\prime}\right) d \omega d v_{*}\right) d v,
\end{aligned}
$$

respectively. In the second and last term of (3.22) we change variables $\left(v, v_{*}\right) \mapsto\left(v_{*}, v\right)$ and $\left(v, v_{*}\right) \mapsto$ $\left(v^{\prime}, v_{*}^{\prime}\right)$, which gives

$$
\begin{aligned}
\iiint_{\mathbb{R}^{3} \times \mathbb{R}^{3} \times S^{2}} B\left(-f^{\prime} f_{*}^{\prime}\left(f+f_{*}-F-F_{*}\right)(f-F)\right) d \omega d v_{*} d v \\
=-\frac{1}{2} \iiint_{\mathbb{R}^{3} \times \mathbb{R}^{3} \times S^{2}} B f^{\prime} f_{*}^{\prime}\left(f+f_{*}-F-F_{*}\right)^{2} d \omega d v_{*} d v \leq 0 .
\end{aligned}
$$

Finally,

Now let

$$
\begin{array}{rl}
\iiint_{\mathbb{R}^{3} \times \mathbb{R}^{3} \times S^{2}} & B f f_{*}\left(f^{\prime}+f_{*}^{\prime}-F^{\prime}-F_{*}^{\prime}\right)(f-F) d \omega d v_{*} d v \\
= & \iiint_{\mathbb{R}^{3} \times \mathbb{R}^{3} \times S^{2}} B f^{\prime} f_{*}^{\prime}\left(f+f_{*}-F-F_{*}\right)\left(f^{\prime}-F^{\prime}\right) d \omega d v_{*} d v \\
= & \iiint_{\mathbb{R}^{3} \times \mathbb{R}^{3} \times S^{2}} B(f-F) f^{\prime} f_{*}^{\prime}\left(f^{\prime}-F^{\prime}\right) d \omega d v_{*} d v \\
& +\iiint_{\mathbb{R}^{3} \times \mathbb{R}^{3} \times S^{2}} B\left(f_{*}-F_{*}\right) f^{\prime} f_{*}^{\prime}\left(f^{\prime}-F^{\prime}\right) d \omega d v_{*} d v \\
= & \int_{\mathbb{R}^{3}}(f-F)\left(\iint_{\mathbb{R}^{3} \times S^{2}} B f^{\prime} f_{*}^{\prime}\left(f^{\prime}+f_{*}^{\prime}-F^{\prime}-F_{*}^{\prime}\right) d \omega d v_{*}\right) d v .
\end{array}
$$

$$
\begin{aligned}
& \Psi_{t}\left(v, v_{*}\right)=f(v, t) f\left(v_{*}, t\right)-F(v) F\left(v_{*}\right), \\
& \tilde{\Psi}_{t}\left(v, v_{*}\right)=f(v, t) f\left(v_{*}, t\right)\left[f(v, t)+f\left(v_{*}, t\right)-F(v)-F\left(v_{*}\right)\right], \\
& \psi\left(v, v_{*}\right)=\int_{S^{2}} B\left(v-v_{*}, \omega\right)\left(1-F^{\prime}-F_{*}^{\prime}\right) d \omega
\end{aligned}
$$

and let

$$
\begin{aligned}
W(t)= & \int_{\mathbb{R}^{3}}(1-F)(f-F) \mathcal{Q}^{+}\left(\Psi_{t}\right)(v) d v-\int_{\mathbb{R}^{3}}(f-F) \mathcal{Q}^{+}\left(\Psi_{t} \mid F\right)(v) d v \\
& +\int_{\mathbb{R}^{3}}(f-F) \mathcal{Q}^{+}\left(\tilde{\Psi}_{t}\right)(v) d v-\int_{\mathbb{R}^{3}} f(f-F)\left(\int_{\mathbb{R}^{3}}\left(f_{*}-F_{*}\right) \psi\left(v, v_{*}\right) d v_{*}\right) d v .
\end{aligned}
$$

Then, omitting the negative term (3.23), we obtain

$$
\begin{aligned}
\int_{\mathbb{R}^{3}} Q(f) & (v, t)[f(v, t)-F(v)] d v \\
& \leq-\int_{\mathbb{R}^{3}}(f-F)^{2}\left(\iint_{\mathbb{R}^{3} \times S^{2}} B F_{*}\left(1-F^{\prime}-F_{*}^{\prime}\right) d \omega d v\right) d v+W(t) .
\end{aligned}
$$

This gives the inequality (3.20).

Step 3: Here we prove that $W(t)$ converges to zero. For this it is enough to check the conditions in Lemma 6 and Lemma 4. First of all we have

$$
(f(\cdot, t)-F) \rightarrow 0 \text { weakly in both } \quad L^{1}\left(\mathbb{R}^{3}\right) \text { and } L^{2}\left(\mathbb{R}^{3}\right) .
$$

The weak $L^{1}$-convergence is a known result, and the weak $L^{2}$-convergence follows directly from this and the $L^{\infty}$-bounds.

Note now that the sets $\mathcal{K}=\left\{\Psi_{t}\right\}_{t \geq 1}$ and $\mathcal{K}=\left\{\tilde{\Psi}_{t}\right\}_{t \geq 1}$ all satisfy the conditions (3.4)-(3.5) in Lemma 4. That follows from the fact that $0 \leq f, F \leq 1$, that $0 \leq \beta \leq 1$, and from the moment estimates which implies that $\sup _{t \geq 1}\|f(t)\|_{L_{s}^{1}}<\infty$ for some $s>2$. 
Also, since $F$ is a Fermi-Dirac distribution (1.2), it is obvious that its Fourier transform $\hat{F}$ belongs to $L^{1}\left(\mathbb{R}^{3}\right)$. Thus by Lemma 4 , the sets $\left\{\mathcal{Q}^{+}\left(\Psi_{t}\right)\right\}_{t \geq 1},\left\{\mathcal{Q}^{+}\left(\Psi_{t} \mid F\right)\right\}_{t \geq 1}$, and $\left\{\mathcal{Q}^{+}\left(\tilde{\Psi}_{t}\right)\right\}_{t \geq 1}$ are all relatively compact in $L^{2}\left(\mathbb{R}^{3}\right)$, and therefore by Lemma 6 the first three integrals in the right hand side of (3.24) tend to 0 as $t \rightarrow \infty$. For the last integral in (3.24), we choose

$$
\begin{aligned}
& P_{t}(v)=f(v, t)(f(v, t)-F(v)) \sqrt{1+|v|^{2}}, \\
& Q_{t}(v)=\int_{\mathbb{R}^{3}}\left(f\left(v_{*}, t\right)-F\left(v_{*}\right)\right) \frac{\psi\left(v, v_{*}\right)}{\sqrt{1+|v|^{2}}} d v_{*} .
\end{aligned}
$$

Since $f$ and $F$ are bounded, and $\|f(t)\|_{L_{2}^{1}} \equiv\left\|f_{0}\right\|_{L_{2}^{1}}<\infty$, it follows that $\left\{P_{t}\right\}_{t \geq 0}$ is relatively weakly compact in $L^{1}\left(\mathbb{R}^{3}\right)$. Also, by definition of $\psi\left(v, v_{*}\right)$ and $0 \leq \beta \leq 1$, we have

$$
\frac{\left|\psi\left(v, v_{*}\right)\right|}{\sqrt{1+|v|^{2}}} \leq A_{0}\left(1+\left|v_{*}\right|^{2}\right)^{1 / 2}
$$

and so by $L^{1}$-weak convergence in (3.25) we have $Q_{t}(v) \rightarrow 0(t \rightarrow \infty)$ for almost every $v \in \mathbb{R}^{3}$. Also it is obvious that $\sup _{t \geq 0}\left\|Q_{t}\right\|_{L^{\infty}}<\infty$. Thus by Lemma 6, the last integral in (3.24) also tends to 0 as $t \rightarrow \infty$. This proves $\lim _{t \rightarrow \infty} W(t)=0$.

The final step is where the high temperature condition comes into play. The game is to prove that there is a constant $c$ such that

$$
\iint_{\mathbb{R}^{3} \times S^{2}} B F_{*}\left(1-F^{\prime}-F_{*}^{\prime}\right) d \omega d v_{*} \geq c \quad \forall v \in \mathbb{R}^{3} .
$$

This would conclude the proof of Theorem 3, because then (3.20) and (3.26) together give the estimate

$$
\frac{1}{2} \frac{d}{d t}\|f(t)-F\|_{L^{2}}^{2} \leq-c\|f(t)-F\|_{L^{2}}^{2}+W(t), \quad t \geq 0
$$

and therefore

$$
\|f(t)-F\|_{L^{2}}^{2} \leq\left\|f_{0}-F\right\|_{L^{2}}^{2} e^{-2 c t}+2 e^{-2 c t} \int_{0}^{t} e^{2 c \tau} W(\tau) d \tau \rightarrow 0,
$$

as $t \rightarrow \infty$, because $W(t)$ converges to 0 as time goes to infinity.

This completes the proof of the strong convergence (3.2), once (3.26) has been established. Now, since the function $\gamma(x)$ is strictly increasing, the formula (1.12) implies that the temperature condition $T \geq$ $\gamma(1) T_{F}$ is equivalent to the condition $a \leq 1$ where $a>0$ is the coefficient in the Fermi-Dirac distribution $F$ for the rescaled number $\varepsilon=1$. Let $\overline{\bar{F}}(r)=a /\left(e^{b r^{2}}+a\right)$. Then, since the mean velocity is zero and $a \leq 1$, we have $F\left(v_{*}\right)=\bar{F}\left(\left|v_{*}\right|\right) \leq 1 / 2$. Because the collisions conserve energy, $\left|v^{\prime}\right|^{2}+\left|v_{*}^{\prime}\right|^{2} \geq\left|v_{*}\right|^{2}$, and it follows that

$$
F\left(v_{*}\right)\left[1-F\left(v^{\prime}\right)-F\left(v_{*}^{\prime}\right)\right] \geq \bar{F}\left(\left|v_{*}\right|\right)\left(\frac{1}{2}-\bar{F}\left(\frac{1}{\sqrt{2}}\left|v_{*}\right|\right)\right):=G\left(\left|v_{*}\right|\right) .
$$

And $G(r)>0$ for all $r>0$. Thus by $\beta \geq 0$ we obtain that for all $v \in \mathbb{R}^{3}$

$$
\begin{array}{rl}
\iint_{\mathbb{R}^{3} \times S^{2}} & B F_{*}\left(1-F^{\prime}-F_{*}^{\prime}\right) d \omega d v_{*} \\
& \geq A_{0} \int_{\mathbb{R}^{3}} G\left(\left|v_{*}\right|\right)\left|v-v_{*}\right|^{\beta} d v_{*} \geq \frac{1}{2} A_{0} \int_{\mathbb{R}^{3}} G\left(\left|v_{*}\right|\right)\left|v_{*}\right|^{\beta} d v_{*}:=c>0,
\end{array}
$$

and hence the the proof of Theorem 3 is complete.

Acknowledgements: The main results were obtained when the first author was visiting Chalmers University of Technology, and he would like to thank Professor Leif Arkeryd for his hospitality. The visit was made possible by a grant from the Wenner-Gren foundation. The research was in part financed by the NSF of China and by the Swedish Natural Sciences Research Fund. 


\section{REFERENCES}

[1] F. Abrahamsson: Strong $L^{1}$ convergence to equilibrium without entropy conditions for the Boltzmann equation, Comm. Partial Differential Equations 24 (1999), no. 7-8, 1501-1535.

[2] R. Alexandre, C. Villani: On the Boltzmann equation for long-range interactions. Comm. Pure Appl. Math. 55 (2002), no. 1, 30-70.

[3] R. Alexandre, L. Desvillettes, C. Villani, B. Wennberg: Entropy dissipation and long range interactions, Arch. Rat. Mech. Anal. 152 no 4, (2000) 327-255.

[4] L. ARKeryd: On the Boltzmann equation, Arch. Rational Mech. Anal. 45 (1972), 1-34.

[5] L. ARKERYD: Intermolecular forces of infinite range and the Boltzmann equation, Arch. Rational Mech. Anal. 77 (1981), no. 1, $11-21$.

[6] Fluid dynamic limits of kinetic equations II, Convergence proofs for the Boltzmann equation, Comm. Pure Appl. Math. 46 (1993), 667-753.

[7] A.V. BobyLEV: Moment inequalities for the Boltzmann equation and applications to spatially homogeneous problems, $J$. Statist. Phys. 88 (1997), no. 5-6, 1183-1214.

[8] F. Bouchut And L. Desvillettes: A proof of the smoothing properties of the positive part of Boltzmann's kernel. Rev. Mat. Iberoamericana 14 (1998), no. 1, 47-61.

[9] E.A. CARLEN AND M.C. CARVAlHo: Entropy production estimates for Boltzmann equations with physically realistic collision kernels, J. Statist. Phys. 74 (1994), no. 3-4, 743-782.

[10] C. Cercignani: The Boltzmann Equation and Its Applications, Springer-Verlag, New York, 1988.

[11] S. Chapman And T. Cowling: The Mathematical Theory of Non-Uniform Gases, Third Edition, Cambridge University Press, 1970 .

[12] J. Dolbeault: Kinetic models and quantum effects: a modified Boltzmann equation for Fermi-Dirac particles, Arch. Rational Mech. Anal. 127 (1994), no. 2, 101-131.

[13] T. Elmroth: Global boundedness of moments of solutions of the Boltzmann equation for forces of infinite range, Arch. Rational Mech. Anal. 82 (1983), no. 1, 1-12.

[14] M. Escobedo AND S. Mischler: On a quantum Boltzmann equation for a gas of photons, J. Math. Pures Appl. (9) 80 (2001), no. 5, 471-515.

[15] M. Escobedo AND S. Mischler: quation de Boltzmann quantique homogne: existence et comportement asymptotique, (French) [Homogeneous quantum Boltzmann equation: existence and asymptotic behavior] C. R. Acad. Sci. Paris Sr. I Math. 329 (1999), no. 7, 593-598.

[16] M. Escobedo, S. Mischler \& M. A. VAlle Homogeneous Boltzmann equation for quantum and relativistic particles, preprint (2002)

[17] T. Goudon: On Boltzmann equations and Fokker-Planck asymptotics: influence of grazing collisions, J. Statist. Phys. 89 (1997), no. 3-4, 751-776.

[18] P.L. LiOns: Compactness in Boltzmann's equation via Fourier integral operators and applications J. Math. Kyoto Univ. 34 (1994), no. 2, 391-427, 429-461.

[19] P.L. Lions: Compactness in Boltzmann's equation via Fourier integral operators and applications III, J. Math. Kyoto Univ. 34 (1994), no. 3, 539-584.

[20] X.G.LU: On spatially homogeneous solutions of a modified Boltzmann equation for Fermi-Dirac particles, J. Statist. Phys. 105 (2001), no. 1-2, 353-388.

[21] X.G.Lu: A modified Boltzmann equation for Bose-Einstein particles: isotropic solutions and long-time behavior, J. Statist. Phys. 98 (2000), no. 5-6, 1335-1394.

[22] X.G. LU: Conservation of energy, entropy identity, and local stability for the spatially homogeneous Boltzmann equation, $J$. Statist. Phys. 96 (1999), no. 3-4, 765-796.

[23] X.G.Lu: A direct method for the regularity of the gain term in the Boltzmann equation, J. Math. Anal. Appl. 228 (1998), no. 2, 409-435.

[24] X.G.Lu AND B. WENNBERG: Solutions with increasing energy for the spatially homogeneous Boltzmann equation, Nonlinear Analysis: RWA 3(2) (2002) 243-258 (to appear).

[25] S. Mischler And B. Wennberg: On the spatially homogeneous Boltzmann equation, Ann. Inst. H. Poincar Anal. Non Linaire 16 (1999), no. 4, 467-501.

[26] R.K. Pathria: Statistical Mechanics, Pergamon Press, 1972.

[27] G. TOSCANI AND C. Villani: Sharp entropy dissipation bounds and explicit rate of trend to equilibrium for the spatially homogeneous Boltzmann equation, Comm. Math. Phys. 203 (1999), no. 3, 667-706.

[28] C. Truesdell And R. Muncaster: Fundamentals of Maxwell's Kinetic Theory of A Simple Monoatomic Gas , Academic Press, New York, 1980.

[29] B. Wennberg: Stability and exponential convergence in $L^{p}$ for the spatially homogeneous Boltzmann equation, Nonlinear Anal. 20 (1993), no. 8, 935-964.

[30] B. Wennberg: Entropy dissipation and moment production for the Boltzmann equation, J. Statist. Phys. 86 (1997), no. 5-6, 1053-1066.

[31] B. Wennberg: Regularity in the Boltzmann equation and the Radon transform, Comm. Partial Differential Equations 19 (1994), no. 11-12, 2057-2074.

[32] C. Villani: On a new class of weak solutions to the spatially homogeneous Boltzmann and Landau equations, Arch. Rational Mech. Anal. 143 (1998), no. 3, 273-307. 
[33] C. Villani: A review of mathematical topics in collisional kinetic theory (2001), to be published in Handbook of Fluid Mechanics, S. Friedlander and D. Serre, Eds.

Department of Mathematical Sciences, Tsinghua University, Beijing 100084, Peoples Republic of China, E-MAIL:XGLU@MATH.TSINGHUA.EDU.CN

Department of Mathematics, Chalmers University of Technology, 41296 Göteborg, Sweden, e-mail: WENNBERG@MATH.CHALMERS.SE 